\title{
Spike history neural response model
}

\section{Tatiana Kameneva, Miganoosh Abramian, Daniele Zarelli, Dragan}

Něsić, Anthony N. Burkitt, Hamish Meffin \& David B. Grayden

Journal of Computational Neuroscience

ISSN 0929-5313

J Comput Neurosci

DOI 10.1007/s10827-015-0549-5

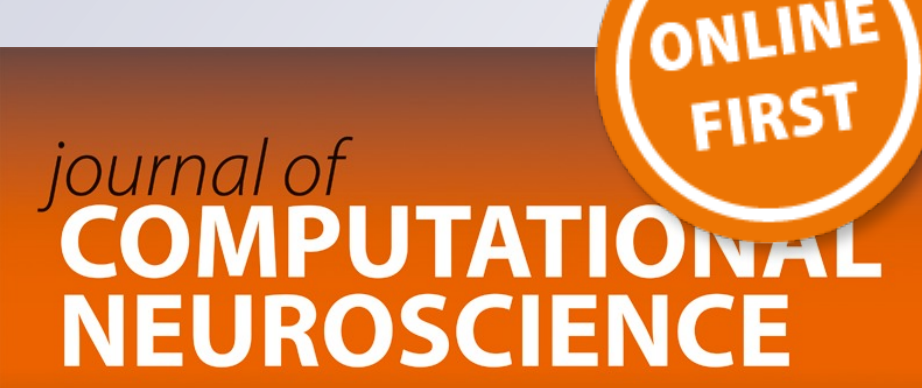

Volume 38 Number 2 April 2015

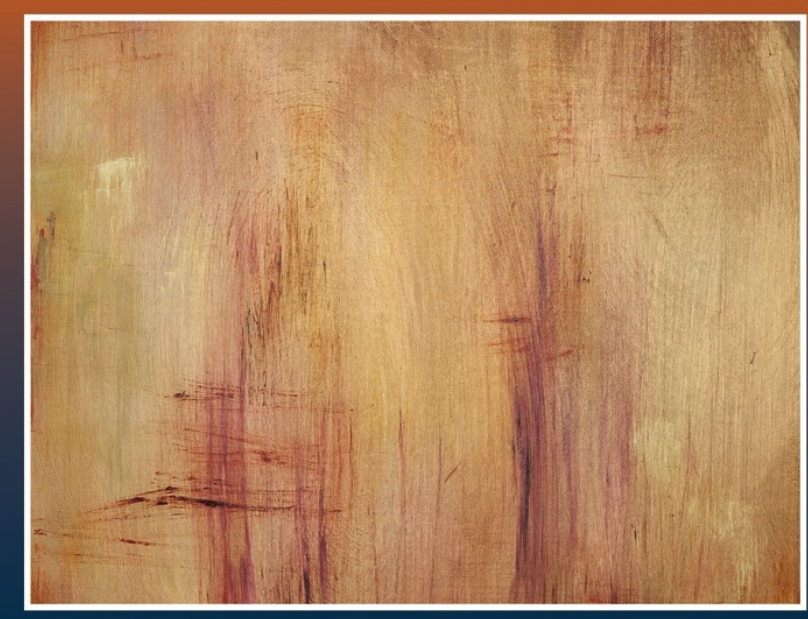

Official Journal of the Organization for Computational Neurosciences

\section{gos}


Your article is protected by copyright and all rights are held exclusively by Springer Science +Business Media New York. This e-offprint is for personal use only and shall not be selfarchived in electronic repositories. If you wish to self-archive your article, please use the accepted manuscript version for posting on your own website. You may further deposit the accepted manuscript version in any repository, provided it is only made publicly available 12 months after official publication or later and provided acknowledgement is given to the original source of publication and a link is inserted to the published article on Springer's website. The link must be accompanied by the following text: "The final publication is available at link.springer.com". 


\title{
Spike history neural response model
}

\author{
Tatiana Kameneva • Miganoosh Abramian • \\ Daniele Zarelli • Dragan Nešić • Anthony N. Burkitt • \\ Hamish Meffin • David B. Grayden
}

Received: 13 August 2014 / Revised: 8 February 2015 / Accepted: 13 February 2015

(C) Springer Science+Business Media New York 2015

\begin{abstract}
There is a potential for improved efficacy of neural stimulation if stimulation levels can be modified dynamically based on the responses of neural tissue in real time. A neural model is developed that describes the response of neurons to electrical stimulation and that is suitable for feedback control neuroprosthetic stimulation. Experimental data from NZ white rabbit retinae is used with a data-driven technique to model neural dynamics. The linear-nonlinear approach is adapted to incorporate spike history and to predict the neural response of ganglion cells to electrical stimulation. To validate the fitness of the model, the penalty
\end{abstract}

Action Editor: Tim Gollisch

T. Kameneva ( $₫)$ · D. Zarelli · D. Nešić · A. N. Burkitt ·

D. B. Grayden

Department of Electrical and Electronic Engineering, University

of Melbourne, Melbourne, Australia

e-mail: tkam@unimelb.edu.au

\section{Abramian}

The University of New South Wales, New South Wales, Australia

A. N. Burkitt · D. B. Grayden

Bionics Institute, East Melbourne, Australia

D. B. Grayden

Victoria Research Laboratory, National ICT Australia,

Melbourne, Australia

H. Meffin

National Vision Research Institute, Carlton, Australia

H. Meffin

Department of Optometry and Vision Sciences, The University

of Melbourne, Melbourne, Australia

T. Kameneva · A. N. Burkitt · H. Meffin - D. B. Grayden

NeuroEngineering Lab, The University of Melbourne,

Melbourne, Australia term is calculated based on the time difference between each simulated spike and the closest spike in time in the experimentally recorded train. The proposed model is able to robustly predict experimentally observed spike trains.

Keywords Retinal ganglion cells $\cdot$ Modeling ·

Simulations · Closed-loop stimulation strategy · Bionic eye

\section{Introduction}

In most currently used neuroprosthetic devices, stimulation parameters are set in the clinic for individual patients but do not change dynamically on a pulse-by-pulse basis in response to the level of the evoked neural activity. While many of these open-loop stimulation strategies are successful (for review, see Clark (2003), Kalia et al. (2013)), the outcomes differ from patient to patient (Cooper and Craddock 2006; Stingl 2013).

In the last decade, there has been increasing interest from the research community and clinicians in implementing closed-loop stimulation strategies in neurobionic devices such as visual prostheses, cochlear implants, and spinal cord stimulation to relieve pain (Abbas and Chizeck 1991; Gorzelic et al. 2013; Fountas et al. 2005; Nelson et al. 2011; Parker et al. 2012; Rosin et al. 2011; Vetter et al. 2010; Zimmermann and Jackson 2014). These schemes adjust stimulation levels dynamically based on the response of neurons in real time. The advantages of the closed-loop strategies include the ability to optimise parameters based on acquired data, to fit parameters based on a specific patient's response, to save power since only the required level of stimulation is delivered to the tissue, and to select and control the stimulation of targeted neural populations (Tukhlina and Rosenblum 2008; Parker et al. 2012). 
To adjust electrical stimulation in a closed-loop bionic device, either a model-independent or a model-based controller design can be implemented. Both techniques have their particular advantages. A model-independent controller does not require knowledge of the system, and consequently it can be used successfully when the prediction of the behaviour of a complex dynamical system is unreliable. To built a model-based controller, a model of the dynamical system is required. Such models, together with simulation tools, may lead to common design environments, rapid prototyping, off-line controller tuning and optimisation, system identification and verification, diagnostics and system self-test. Model-based controller design has the following steps: (i) modeling a system, (ii) designing and analyzing a controller for the system, (iii) simulating the system and controller, and (iv) integrating all these steps by implementing the controller in a physical system.

In this work, we focus on the first three steps of controller design. There are two main techniques for system modeling: data-driven or built on first principles. The data-driven techniques use system identification theory tools to analyze data from a system, and choose and implement algorithms that can identify a mathematical model underlying the realworld system. The first principles techniques are founded on representation of the physical elements of the actual system and their dynamics by differential-algebraic equations.

In the case considered here, we collect electrophysiological data experimentally from retina slices and use a data-driven technique to model neural dynamics. Our motivation comes from visual prostheses. It has been shown that it is possible to elicit small spots of light (called phosphenes) by electrically stimulating surviving neurons in the retina in people with retinitis pigmentosa and age-related macular degeneration. A mosaic of phosphenes can provide perception of a two-dimensional image (Stingl 2013).

Models suitable for the controller design need to have some specific characteristics. The feedback signal that is provided to the controller must be possible to measure in vivo. For example, to measure a membrane potential is impractical due to the limited bandwidth for power and data transfer in bionic devices, and due to the impossibility of recording intracellular signals. However, measuring the average spiking rate of a population of neurons surrounding the recording electrode is plausible. The models should be parametrizable for different types of neurons or population of neurons. The models need to be tractable and simple enough so that the calculation of the controller signal can be done in real time without requiring high performance computational facilities. While there exist many models that describe neural response to sensory or electrical stimulation (for review, see Dayan and Abbott 2001; Koch and Segev 1998), not all of them can feasibly be used for the controller design.
An example of a model that can be used for the controller design is a linear-nonlinear model (Chichilnisky 2001). This model has been successfully applied in many applications, including to describe the response of retinal ganglion cells (RGCs) to light stimulation. The model involves two stages. The first, linear stage is a spatial and temporal filtering of the visual stimulus, which models the synaptic filtering that gives rise to the receptive field properties of the RGC. Physiologically, the filter may be interpreted as the receptive field of the neuron, and the filter output may be interpreted as the subthreshold membrane potential prior to the activation of voltage-gated ion channels associated with action potential initiation. The second, nonlinear stage accounts for spike generation by applying a nonlinear threshold function to the output for the first stage. This captures the neural spike rate as a function of depolarizing drive: the drive must reach a minimum threshold before a spike occurs; above this level, increased drive leads to an increasing spike rate up to some saturation level.

A key advantage of this form of model is that there are well-established experimental protocols for accurately estimating the linear filters and the nonlinear function using reverse correlation and maximum likelihood methods (Chichilnisky 2001; Lesica et al. 2007; Nirenberg et al. 2010). Briefly, the simplest of these methods involves recording the RGC's response to a long sequence of pixelised images, in which the brightness of each pixel is chosen randomly at every frame from a Gaussian distribution. The spatio-temporal filter corresponding to the neuron's receptive field is recovered by calculating the cross-correlation in time between the recorded spike train and the sequence of pixelised image frames. The nonlinearity is recovered by creating a histogram of the number of spikes that occurred as a function of the filtered output value. Alternative methods that maximise the likelihood of predicting the recorded spike train are based on optimisation techniques and allow the linear-nonlinear model to be fitted to data based on stimuli other than Gaussian white noise, such as natural scenes (Nirenberg et al. 2010). Together, these models and methods have demonstrated a remarkable ability to predict the response of RGCs to a large variety of stimuli (Chichilnisky 2001; Lesica et al. 2007; Nirenberg et al. 2010). These linear-nonlinear models are often referred to as "generalized linear model" (Pillow et al. 2005).

The limitation of the linear-nonlinear model is that it does not account for the history of the response of the neuron itself. It has been shown that RGCs typically respond with brief clusters of spikes separated by a period of silence in response to naturalistic movies, random visual flicker, and white noise visual stimulation (Chichilnisky 2001; Keat et al. 2001; van Hateren et al. 2002). The phenomena of spike clusters separated by periods of suppression is 
characteristic of a memory in the system; i.e., the response of the neuron depends on its previous response. To describe such phenomena, a model that incorporates spike history is usually required. Our modeling technique is based on the linear-nonlinear method described above but also incorporates spike history into the model.

The work presented here was carried out in three steps. All steps were done keeping in mind that the model has to be suitable for the controller design. First, we designed a protocol and collected experimental data that could be used for our particular modeling set-up. Then, we designed a model and fitted modeling parameters to the experimental data. Last, we simulated response of the RGCs to electrical stimulation. Validation of the controller design in vitro and in vivo will be carried out in a future study.

\section{Methods}

\subsection{Experiments}

Experimental data were collected to build a neural response model to use in a closed-loop stimulation paradigm.

\subsubsection{Retinal preparation}

In vitro experiments were conducted at the University of New South Wales (UNSW) on NZ White rabbit (2.5-3.5 kg) retina preparations. In total, data from 7 cells were collected, some of which were repeatedly stimulated with the stimulation protocols described below.

All procedures were approved by the UNSW Animal Care and Ethics Committee. To anaesthetize the rabbits, Isoflurane ( $4 \%$ in $\mathrm{O}_{2}, 2 \mathrm{~L} / \mathrm{min}$ ) was delivered through a mask. Subsequently, one eye was enucleated and the animal was immediately euthanized by lethal overdose of sodium pentobarbital $(150 \mathrm{mg} / \mathrm{kg})$. The eye was placed in sodium bicarbonate buffered ( $\mathrm{pH}$ 7.4) Ames medium (Sigma Aldrich), which was continuously bubbled with $95 \% \mathrm{O}_{2}$ and $5 \% \mathrm{CO}_{2}$ at room temperature. A $5 \mathrm{~mm}$ by $10 \mathrm{~mm}$ piece of inferior retina with attached sclera, including the optic nerve head, was excised. The tissue was placed flat, RGC side up, in a perfusion chamber (Warner instruments) and was held in place using a slice anchor (Warner Instruments). It was perfused at a rate of $4-5 \mathrm{~mL} / \mathrm{min}$ with oxygenated Ames medium ( $\mathrm{pH} 7.4,36 \mathrm{degC}$ ).

\subsubsection{Stimulating electrodes}

Stimulating electrodes were made from Teflon-coated platinum wires (A-M Systems Inc, WA), which were cut so that the disc-shaped platinum cross-section was exposed. Seven wires were arranged hexagonally using a 7-barrel glass tube (World Precision Instruments), which was heated and pulled with a glass micro-pipette puller. These electrodes were used in a hexapolar configuration, where the stimulus pulse was delivered via the electrode at the centre of the hexagon and returned via the six guard electrodes located on its vertices. The electrodes were $50 \mu \mathrm{m}$ in diameter and their center-to-center spacing was $200 \mu \mathrm{m}$.

\subsubsection{Stimulating and recording setup}

Stimulating electrodes were mounted on a three-axis motorized micromanipulator (MP-285 Sutter Instruments), programmed to perform automated translation. Single-unit extracellular recordings were obtained from RGC somas with a pair of $1 \mathrm{M} \Omega$ tungsten microelectrodes (A-M Systems Inc, WA), which were mounted on a one-axis motorized micromanipulator (PCS 4100, Burleigh). The tip-to-tip separation between the micro-electrodes was $200 \mu \mathrm{m}$ for the closest, up to $500 \mu \mathrm{m}$ for the distant. RGC spikes were recorded in differential mode using a battery powered differential amplifier (DAM80, World Precision Instruments). The recorded signals were amplified 10,000 times and bandpass filtered between $0.3 \mathrm{kHz}$ and $3 \mathrm{kHz}$. A custom-written LabVIEW (National Instruments, version 10) program was used for delivering the stimulus pulse trains as well as recording the RGC responses at sampling rate of $20 \mathrm{kHz}$ (USB-6211 DAQ, National Instruments).

\subsubsection{Experimental protocol}

Experiments were performed under dim red light within a Faraday cage, which was positioned on an anti-vibration table. To record RGC action potentials, the recording microelectrode pair was lowered onto the retinal nerve fibre layer. Proper placement of the microelectrodes was confirmed by recording spontaneous and light-evoked RGC spikes. The recording electrodes were typically $>5 \mathrm{~mm}$ inferior to the optic nerve head, while the stimulating electrodes were positioned at least 1-2 mm superior to the recording electrodes, along the inferred axonal path. Supra-threshold current pulses were delivered through the hexapolar electrodes. If no electrically evoked responses were recorded, the stimulating electrodes were moved perpendicular to the inferred axonal path in $100 \mu \mathrm{m}$ increments. To ensure that the retinal tissue was not dragged or damaged, the stimulating electrodes were always lifted $1 \mathrm{~mm}$ above the retinal surface before altering the lateral position. The electrode position on the retinal surface where the activation was achieved with lowest amount of stimulus current was chosen as the stimulation site and remained unchanged for all subsequent measurements. Only single-cell RGC responses were studied, which were all-or-none spikes and exhibited 
near-constant latency, amplitude, and shape. Single-unit spikes were readily distinguishable from multi-unit recordings, which were generally multi-peak signals and exhibited variable shape and amplitude. These signals were a result of recording electrodes being in close proximity to a number of simultaneously active RGCs. The position of the recording electrodes almost always had to be adjusted to isolate activities of a single RGC. After a cell response was successfully isolated, it was stimulated with trains of white noise pulses as described below.

\subsubsection{White noise stimulation}

Electrical stimulation consisted of a train of bi-phasic pulses of equal phase duration of $100 \mu$ s per phase and zero interphase gap. Each stimulation pulse train consisted of 10000 bi-phasic pulses. The amplitude of the pulse was selected randomly at each stimulation time from a Gaussian distribution with zero mean and variance $\sigma^{2}$. This distribution was generated in MATLAB $^{\odot}$ (Mathworks, version 2011) with zero mean and $\sigma=1$; stimulation amplitudes above $4 \sigma$ were clipped at $4 \sigma$. The generated pulse train with amplitudes on the interval $[-4 \sigma, 4 \sigma]$, was mapped to the interval [ $\left.-A_{\max }, A_{\max }\right]$, were $A_{\max }$ is the maximum stimulation amplitude used in simulations. This allowed oversampling at the lower amplitude levels (when there were not enough spikes to obtain sufficient statistics), and less sampling at the higher amplitude levels (when cells spike often). The value of $A_{\max }$ is discussed below. Cathodic-first and anodic-first pulses were both used in the stimulation train, corresponding respectively to positive and negative values of the amplitude.

\subsubsection{Stimulation protocol 1}

To explore differences in responses of RGCs to different frequencies of stimulation and to explore if there is memory in the RGC's responses, a set of experiments was conducted where the white noise pulse train was delivered at $25,50,100,200,500$, and $1000 \mathrm{~Hz}$, with $A_{\max }=25,50$, and $100 \mu \mathrm{A}$. In total, 4 cells were stimulated using this protocol.

\subsubsection{Stimulation protocol 2}

The results of the experiments with Protocol 1 revealed clumping of the evoked spikes at stimulation frequencies higher than $100 \mathrm{~Hz}$; i.e., clusters of spikes occurred together with periods of suppression (no spiking) between clusters. To further explore this effect, a second set of experiments were conducted where the stimulus frequency was set to 200 $\mathrm{Hz}$. For each cell, several repetitions (6-10, depending on the cell's health) of the same stimulation were carried out to examine how well the model fitted the experimental data, i.e., robustness of the model parameters to the repeated stimulation. A recovery time of up to one minute was allowed in between repetitions to ensure that responses to consecutive stimulations were not influenced by the previous stimulus trains. The maximum amplitude of the white noise train, $A_{\max }$, was adjusted for each cell and was set to twice the average threshold measured for that cell, as defined in the following section. In total, 3 cells were repeatedly stimulated with this protocol.

\subsubsection{Threshold measurements}

Stimulus current pulses were applied to the retinal surface starting at a sub-threshold level. The amplitude was then increased step-wise in $0.2-2.0 \mu \mathrm{A}$ increments. At each amplitude, RGC responses to 10 consecutive pulses were recorded and the number of pulses that evoked an action potential was determined. A sigmoid curve was then fitted to the data (GraphPad Prism 5 software). The pulse amplitude required for evoking cell responses $50 \%$ of the time was estimated from the curve fit and taken as the threshold. Thresholds were measured for the case of anodic-first (A) and cathodic-first (B) biphasic pulses, and the average threshold, $(\mathrm{A}+\mathrm{B}) / 2$, was calculated.

\subsection{Modeling}

Experimental data were used to characterize the response properties of neurons to the electrical stimulation. The linear-nonlinear approach, widely used to predict neural response to light stimulation (Dayan and Abbott 2001, Jepson et al. 2014a), was adapted to predict neural response of RGCs to electrical stimulation. In contrast to traditional linear-nonlinear schemes, the model proposed here incorporates the history of the response of a neuron. To emphasize this feature of the model, we call it the "spike history model".

To estimate the responses of cells to electrical stimulation, we used a novel pseudo-random electrical stimulation. In particular, we used modified Gaussian white noise electrical stimulation, where the amplitude of a biphasic pulse was drawn from a Gaussian distribution with zero mean, as described in Section 2. An example of Gaussian white noise pulse stimulation is shown in Fig. 1. In this example, $t_{n}$ is time, $n=1,2, \ldots, s_{n}$ is a stimulus applied at the time $t_{n}$, and $r_{n}$ is a response of a neuron at time $t_{n}$. To estimate the response of a neuron at the time $t_{17}$, the stimulus at the time $t_{16}, s_{16}$, and the response on the time interval $t_{11} \leq t \leq t_{16}, r_{[11,16]}$, were used. In the above, $t_{n}$ are the sampling times. The start of each sampling interval is immediately after the end of the stimulation pulse, as illustrated in Fig. 1. Therefore, the response at the time $t_{n}$ depends on 


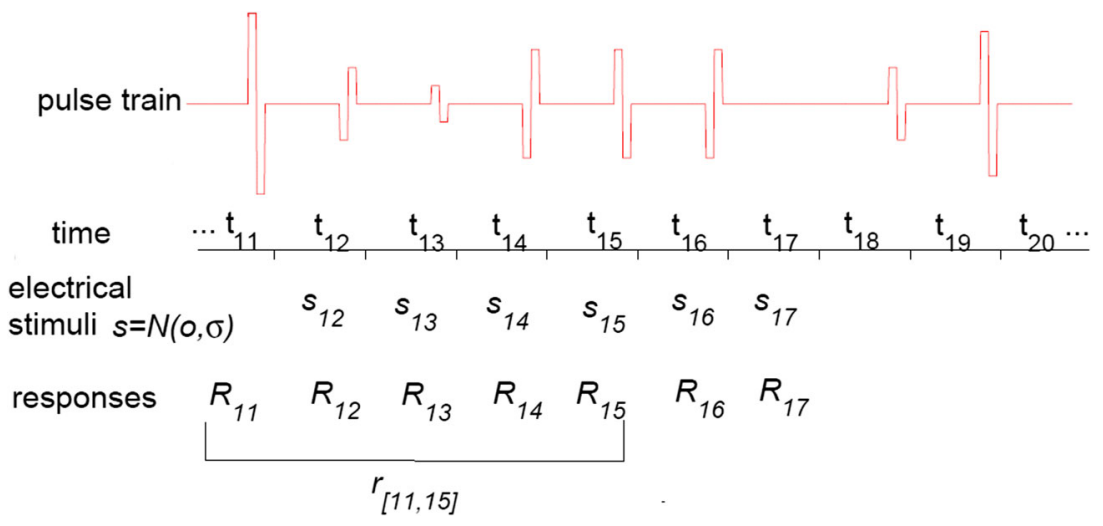

Fig. 1 An example of Gaussian white noise biphasic pulse stimulation. An example of the bi-phasic electrical pulse sequence is shown at the top, the sampling times (time bins) are shown immediately below. Electrical stimuli are defined by $s_{n}$, neural responses are defined by

the pulse amplitude at $t_{n-1}$. Note, we recorded only direct responses of RGCs, and at most one spike was recorded in each time bin.

We propose the following formula to estimate the conditional probability of spiking $\left(r_{t}=1\right)$ at the time $t$ given a stimulus, $s_{t-1}$, and a response history, $r_{\left[t-t_{\mathrm{m}}, t-1\right]}$ :

$$
\begin{aligned}
P_{\text {spike }}\left(r_{t}=1 \mid s_{t-1}, r_{\left[t-t_{\mathrm{m}}, t-1\right]}\right)= & P_{t}=G\left(a s_{t-1}+b\left|s_{t-1}\right|\right. \\
& \left.+h \cdot r_{\left[t-t_{\mathrm{m}}, t-1\right]}\right),
\end{aligned}
$$

where $a, b$ are the response constants measured in units $\mu A^{-1}$, required to scale the anodic and cathodic responses differently, $h$ is a dimensionless spike history kernel, $s_{t-1}$ is the amplitude of the first phase of the bi-phasic pulse at the time $t-1, r_{\left[t-t_{\mathrm{m}}, t-1\right]}$ is the response on the time interval $t-t_{\mathrm{m}} \leq t \leq t-1$ that is dimensionless (measured in zeros (no spike) and ones (a spike)), $t_{\mathrm{m}}$ is a duration of the memory in the system. $P_{t}$ is dimensionless. We have chosen this structure of the model, because of the success of existing linear-nonlinear models (Jepson et al. 2014a).

$G$ is the neuron's nonlinearity function. We assume that $G$ can be approximated by a sigmoid, due to existing experimental data that shows this input-output relationship (Gerstner and Kistler 2002),

$G(x)=\frac{1+\tanh [x-d]}{2}$,

where $d$ is a single pulse threshold measured in units of $\mu A$. The argument of $G$,

$x=a s_{t-1}+b\left|s_{t-1}\right|+h * r_{\left[t-t_{\mathrm{m}}, t-1\right]}$

is called a generator signal. To have a bandpass response in time, $h$ can be approximated by a double exponential function,

$h(t)=A_{1} e^{-t / \tau_{1}}+A_{2} e^{-t / \tau_{2}}$.

$A_{1}, A_{2}$ are the dimensionless kernel's amplitude parameters that will have opposite sign, $\tau_{1}, \tau_{2}$ are time constants
$R_{n}, n=12,13, \ldots$ In this example, to calculate the neural response at time $t_{17}$, the stimulation amplitude, $s_{16}$, and the vector of the previous responses, $r_{[11,15]}$, were used. The memory of the system in this example is five time bins

measured in ms. Note, the model predicts one type of kernel and sigmoid irrespective of the cathodic- or anodic-first biphasic pulse used. (This is a realistic assumption since the difference between cathodic- and anodic-first pulse threshold was less than $10 \%$ in our experiments, as illustrated in Fig. 11a.)

The predicted probability of a spike at time $t$ was calculated as

$P_{t}=G$ if a spike occured at time $t$,

and the predicted probability of no spike at time $t$ as,

$\bar{P}_{t}=1-P_{t}=1-G$ if no spike occured at time $t$.

In the last step, the log-likelihood, $L$, of predicting the experimentally observed spike train from one stimulus pulse to the next, given the previous stimulus and spike history, is maximized.

Let $S=\{t \mid$ there was a spike recorded at $t\}$ be the set of all time bins in which a spike was recorded.

Let $\bar{S}=\{t \mid$ there was no spike recorded at $t\}$ be the set of all time bins in which no spike was recorded.

Then,

$L=\sum_{S} \log \left(P_{t}\right)+\sum_{\bar{S}}\left(1-\log \left(P_{t}\right)\right)$.

To fit model parameters to the experimental data, the parameters $a, b, d, A_{1}, A_{2}, \tau_{1}$, and $\tau_{2}$ were optimized to maximize the log-likelihood, $L$, for all stimulus presentations and recorded spike trains. The optimization was performed in MATLAB ${ }^{\odot}$, using the nonlinear least squares problem solver, lsqnonlin. Levenberg-Marquardt algorithms was used with tolerance level for the function and argument of $10^{-7}$. The following initial values were used: $a=$ $3 \mu A^{-1}, b=33 \mu A^{-1}, \tau_{2}=3333 m s, d=0$. Initial 
values for $A_{1}, A_{2}$ were set proportional to the number of successful stimuli in the stimulation train: $A_{1}=13333 *$ $N / N_{\text {spikes }}, A_{2}=-6666 * N / N_{\text {spikes }}$, where $N=10000$ is the number of pulses and $N_{\text {spikes }}$ is the number of evoked spikes in the experimentally recorded spike train.

To convert probability into a stochastically predicted spike train, a Poisson process was used: a spike was placed in a time bin (at a sampling interval), if the predicted probability, $P_{t}$, was larger than a randomly generated number from the standard uniform distribution on the open interval $(0,1)$.

To compare the model (1) with the model without history, we also calculated the probability of spiking using the formula without the response kernel,

$P_{t}^{\not h}=G\left(a s_{t-1}+b\left|s_{t-1}\right|\right)$

and computed Eqs. (2)-(7) for $P_{t}^{\not h}$.

To compare how well the simulated spike train approximated experimentally recorded spikes, we performed the following analysis. Let $\Delta_{i}$ be the magnitude of the time difference between each model spike and the closest spike in time in the experimentally recorded train, clipped at time five bins, measured in ms. Let $N_{s}$ be the total number of spikes in the simulated spike train. Then, the closeness of the experimentally recorded and modelled spike trains is approximated by

$E_{\Delta}=\sum_{i=1}^{N_{\mathbf{s}}} \Delta_{i} f$

where $f$ is the frequency of stimulation in cycles per ms. The error, $E_{\Delta}$, is dimensionless; it is a simple measure to account for the spike insertions and deletions in the modelled spike train. The lower the value of $E_{\Delta}$, the better the model approximates the experimental data. $E_{\Delta}=0$ when all modelled spikes are in the correct sampling intervals (or when there are no spikes in the simulated spike train). We calculate the value of $E_{\Delta}$ for the spike history model (1) and for the model without the response kernel (8).

The metric-space analysis of a spike train allows comparison of the temporal coding properties of the simulated train with the experimentally recorded one (Victor and Purpura 1997). The Victor-Purpura metric has a penalty unit cost for each spike insertion or deletion. The metric based on the spike times has a penalty cost $q d t$ for each simulated spike time shifted by $d t$ from the recorded spike time. The metric based on the inter-spike intervals has a penalty cost $q d \tilde{t}$ for each shifted simulated inter-spike interval by $d \tilde{t}$ in comparison with the recorded spike time. The parameter $q$ has units $\mathrm{s}^{-1}$, it expresses the sensitivity of the metric to temporal pattern, and represents a relative cost of moving a spike or changing the duration of the interval. For $q=1$, the metric based on the spike times is equal to $E_{\Delta} / f$. For $q=\infty$, the spike times and inter-spike interval metrics depends only on the number of spikes and are equal. We calculated the metrics for $q=1, q=10$, and $q=100$, and compare these for the model with the spike history and for the model where the spike history is neglected.

In addition, we compared the values of the coefficient of variation of the interspike intervals for the experimentally recorded spike train, for the simulated spike train with the history model (1), and for the modelled spike train where the spike history is neglected (8).

To investigate whether the data showed spike history phenomena, we calculated the autocorrelation function, $w$, at each sampling time (at each pulse instance),

$w(k)=\sum_{j} r_{j} \cdot r_{k+j+1}$,

where $r_{j}$ is the response at time $j$ (zero or one).

\section{Results}

The evoked responses were generally time-locked to the stimulus pulses, $3 \mathrm{~ms}$ after the stimulation. The observed latencies of several milliseconds were due to the time required for the action potential to travel along the axon and arrive at the recording site. Both the stimulating and the recording electrodes were fixed in place during stimulation of the cell and, therefore, any changes in the relative latencies were most likely caused by the stimulus parameters. In other words, the absolute latencies were not intrinsic properties of the cell responses but relative changes in the latencies were.

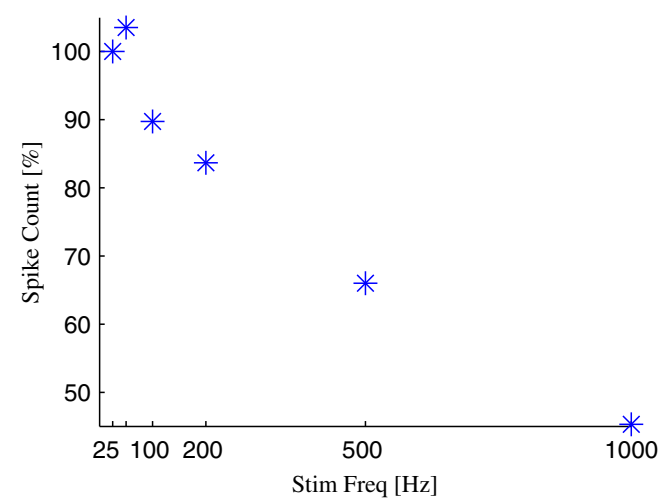

Fig. 2 Experimental data using Protocol 1. Spike count as a function of stimulation frequency. Stars show averaged data for each stimulation frequency normalized to $100 \%$ at $25 \mathrm{~Hz}$ stimulation. $50 \mathrm{~Hz}$ stimulation frequency is indicated by a tick only for better visualization 
Fig. 3 Examples of experimentally recorded spike trains. Dots represent experimentally recorded spikes. The top two spike trains are shorter than others due to the shorter end of the stimulation time for the high frequency of stimulation (corresponded to 5000 biphasic pulses)

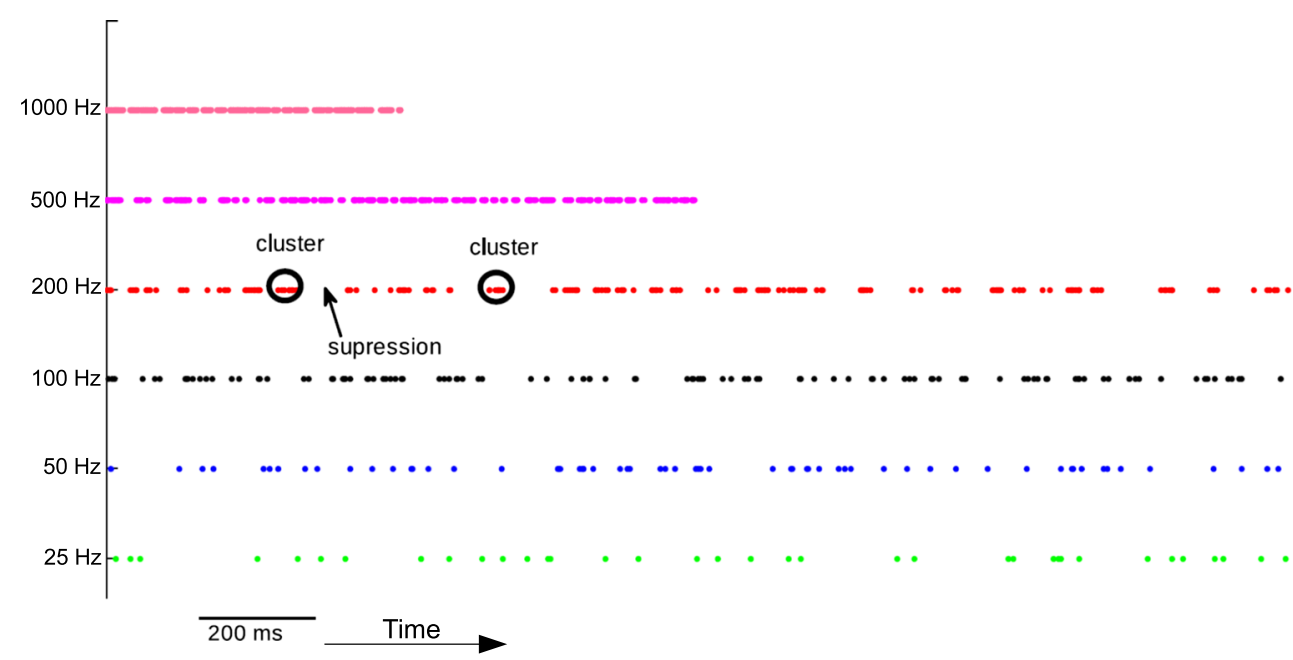

to $50 \mu \mathrm{A}$ and then to $25 \mu \mathrm{A}$, as a common technique used for the higher frequency stimulation.

All cells responded to all frequencies of stimulation. A decrease in spike count was observed with increased frequency of stimulation. Figure 2 illustrates experimental data averaged for each frequency of the stimulation, normalized to $100 \%$ at $25 \mathrm{~Hz}$ stimulation.

An example raster plot of the responses of the cells to different frequencies of stimulation is shown in Fig. 3. A strong
Fig. 4 Autocorrelation function for different frequencies of stimulation plotted as a function of the pulse number. The same cell is stimulated with $25 \mathrm{~Hz}$ $200 \mathrm{~Hz}$ frequencies, a different cell is stimulated with $500 \mathrm{~Hz}$ and $1000 \mathrm{~Hz}$ frequencies. The frequency of the stimulation is denoted above the subplot
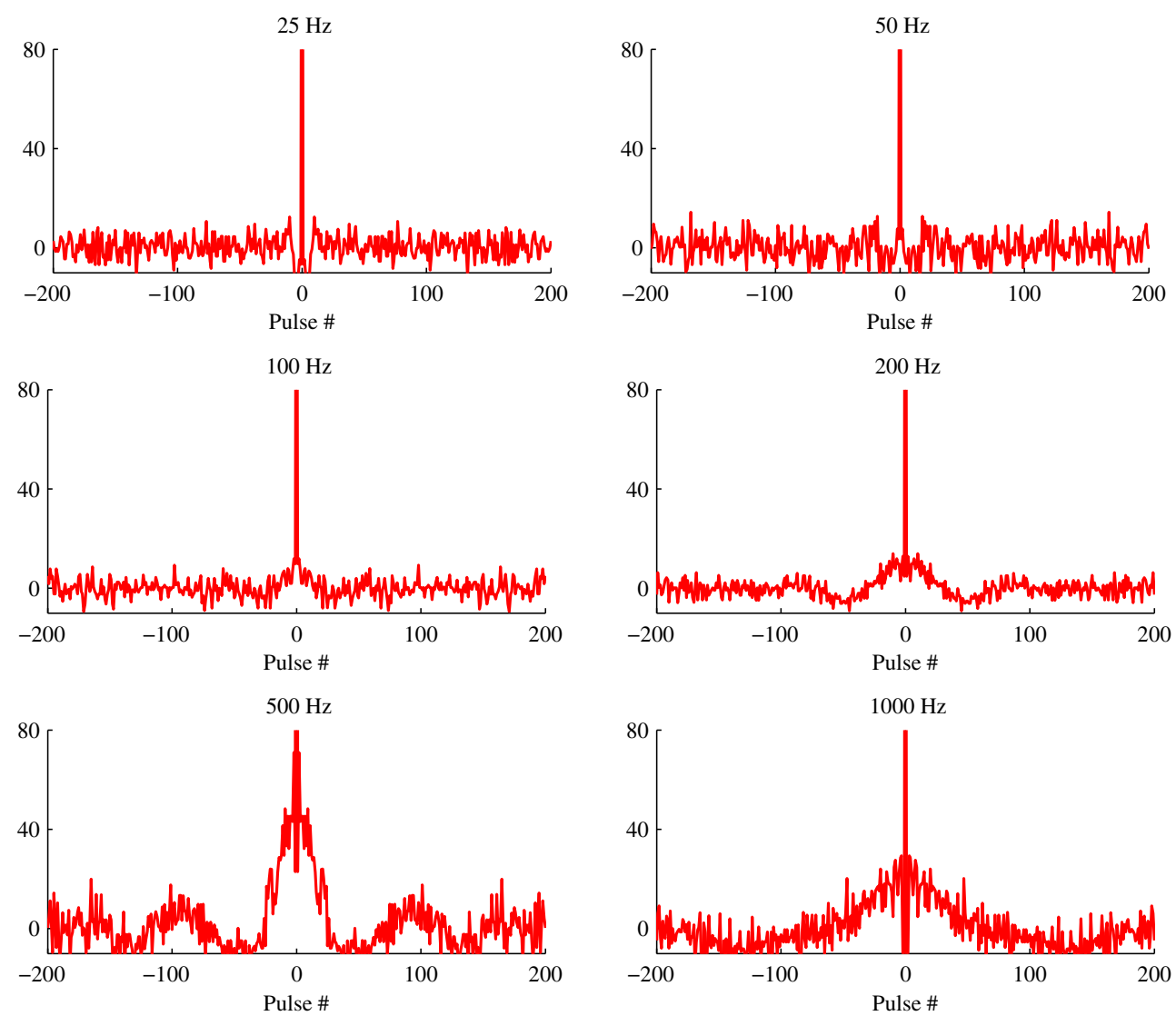
Fig. 5 Autocorrelation function for different frequencies of stimulation plotted as a function of time. The same cell is stimulated with $25 \mathrm{~Hz}-200 \mathrm{~Hz}$ frequencies, a different cell is stimulated with $50 \mathrm{~Hz}$ and 1000 $\mathrm{Hz}$ frequencies. The frequency of the stimulation is denoted above the subplot
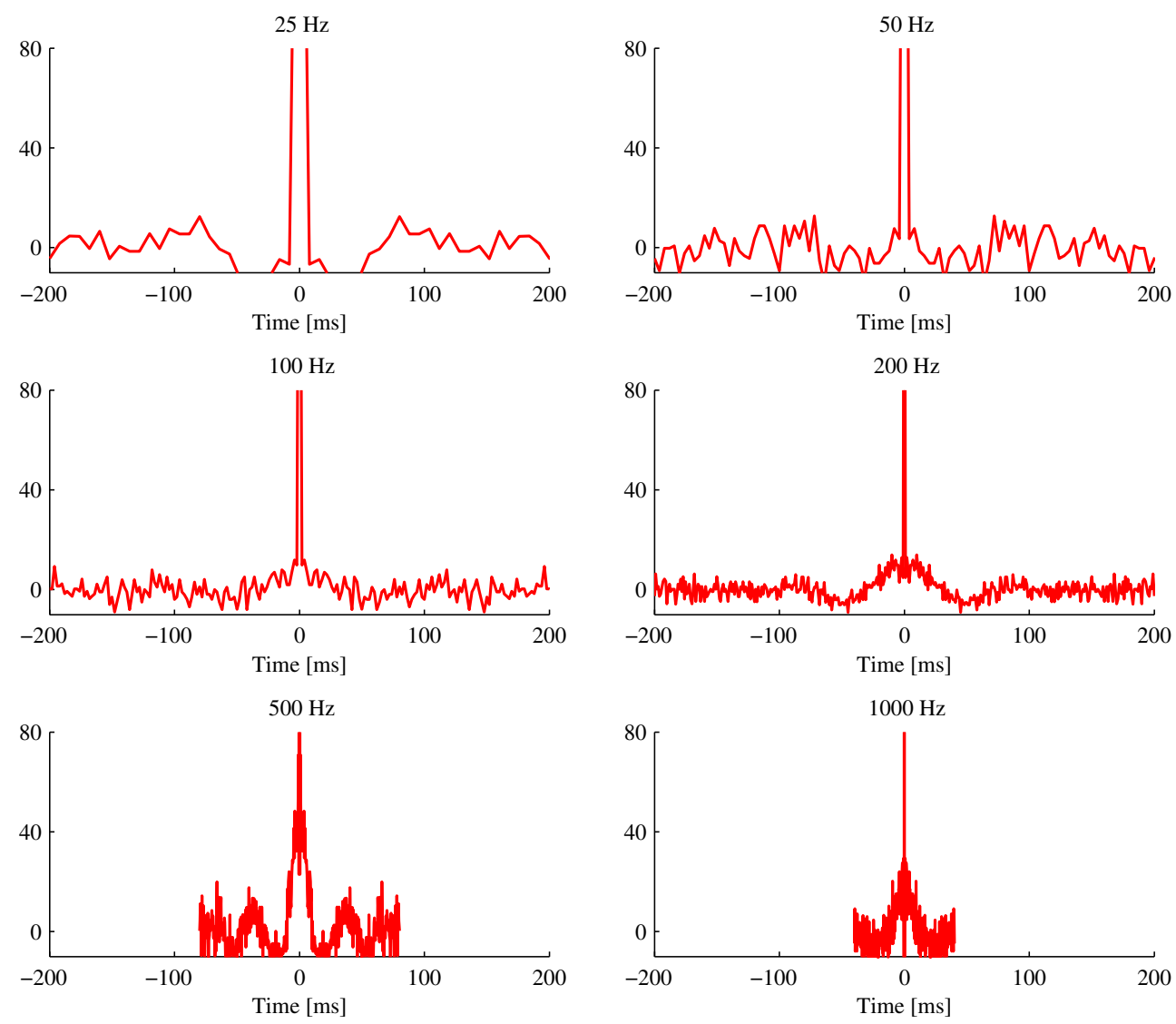

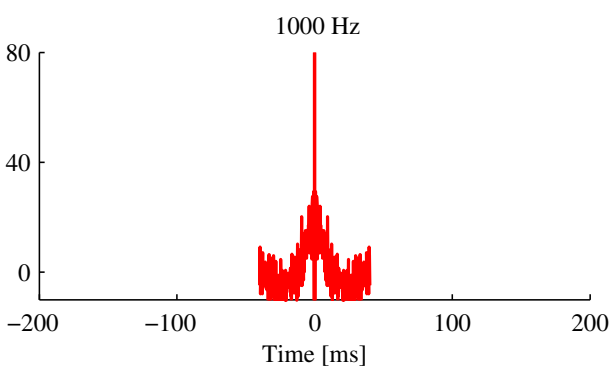

effect of spike clumping is observed at $200 \mathrm{~Hz}$ stimulation. Ovals show examples of a cluster of spikes.

The phenomenon of a cluster-suppression response to white-noise stimulation may be indicative of memory in the system. That is, the response depended not only on the current stimulus but also on the response in some time window preceding the current time. To confirm this, we plotted the auto-correlation function of the recorded spike trains for different frequencies of stimulation, as illustrated in Figs. 4 and 5. Given the stimulus was white noise and that each pulse had a certain probability of causing a spike, independent of the other pulses, the auto-correlation function should be a delta function on a baseline of noise if there is no memory in the system. This is not the case for stimulation at frequencies higher than $100 \mathrm{~Hz}$. Instead, there appears to be some non-zero correlation around the origin. This is consistent with the way that the recorded spikes tend to clump together as shown in Fig. 3 for $200 \mathrm{~Hz}$ (red). Figure 5 shows that the effect of memory is noticeably on the longer time scale for $100 \mathrm{~Hz}$ and $200 \mathrm{~Hz}$ (see the slopes on both sides on the delta function at zero $\mathrm{ms}$ ), than for other frequencies of stimulation. In order to limit the spontaneous activity interference with the electrical response properties, the cells with low spontaneous activity were targeted for the experimental recordings. Due to the limited number of spontaneous spikes, the autocorrelation function would represent mostly noise in this case. Therefore, the autocorrelation functions shown in Figs. 4 and 5 cannot be compared with the autocorrelation function of non-stimulated neurons.

After the initial experiments with Protocol 1, all subsequent experiments were conducted only at $200 \mathrm{~Hz}$ stimulation. While the effect of clumping was observed at 500 $\mathrm{Hz}$ and $1000 \mathrm{~Hz}$ stimulation, it was unreasonable to expect that the cell would be able to follow such high frequencies of stimulation when the same stimulation pulse train was applied up to 10 times for a prolonged period of time of each trial.

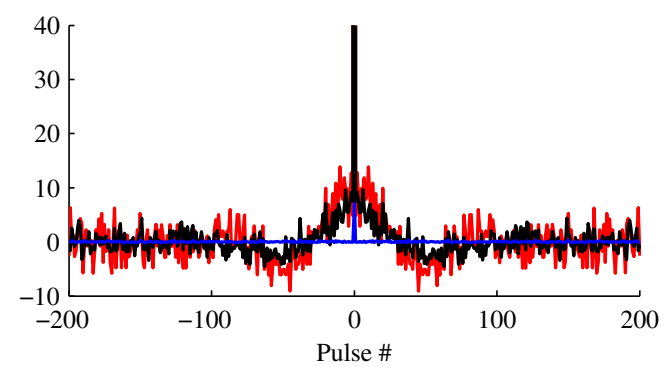

Fig. 6 Auto-correlation function for the recorded spike trains (red), for the model with history (black) and the model without history ( $h=$ 0, blue) for $200 \mathrm{~Hz}$ stimulation 
Fig. 7 The model predictions vs. recorded spike trains for six different pulse frequencies. Red dots indicate experimentally recorded spikes, blue lines give the predicted spike probabilities for pulses, and black dots give a stochastically predicted spike train based on the spike probability. Data from the middle of experiments, for 1400 pulses, are shown a) $25 \mathrm{~Hz}$

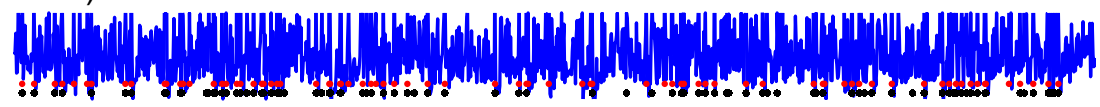

b) $50 \mathrm{~Hz}$

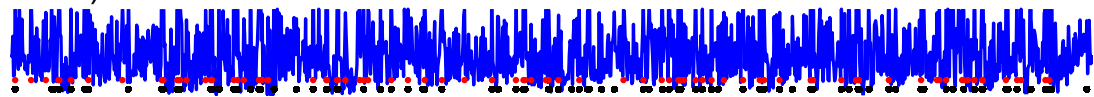

C) $100 \mathrm{~Hz}$

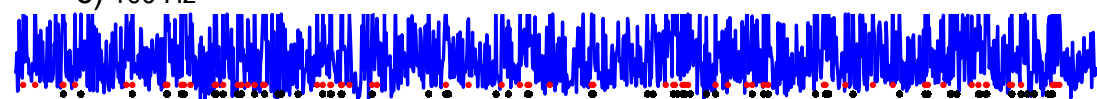

d) $200 \mathrm{~Hz}$

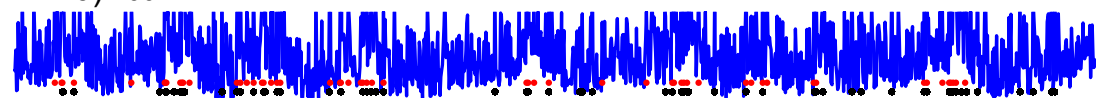

e) $500 \mathrm{~Hz}$

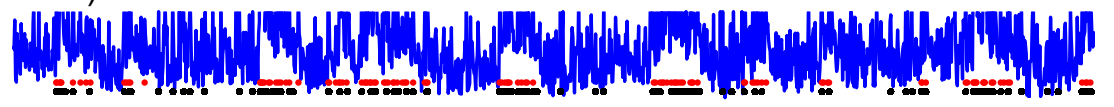

f) $1000 \mathrm{~Hz}$

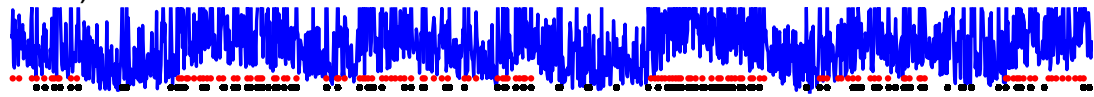

Pulse number

200

\subsubsection{Modeling}

The auto-correlation function is useful to consider because it can show whether there are some dynamics that are not captured by the model. Figure 6 shows the auto-correlation function of the recorded spike trains (red), the model with history (black), and the model without history (blue) for $200 \mathrm{~Hz}$ stimulation. The figure illustrates that the auto-correlation function of the model with the response kernel approximates the auto-correlation function of the
Fig. 8 a) Nonlinearity $G$, calculated for different frequencies of stimulation for the same cell, plotted as a function of generator signal, $x$. b) Response kernel, $h$, calculated for different frequencies of stimulation for the same cell; plotted as a function of a pulse number in (b) and as a function of time in (c) a)

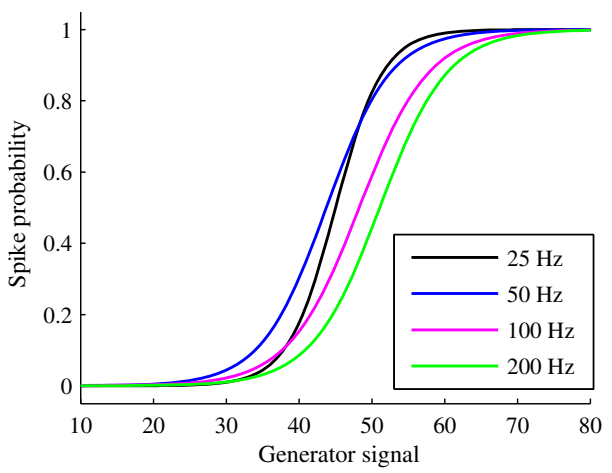

c) b)

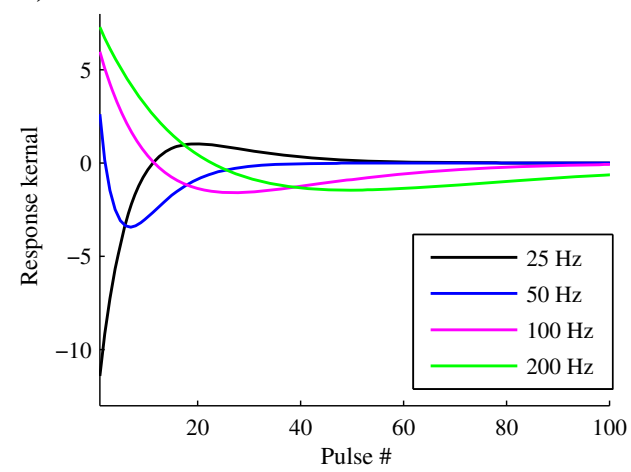

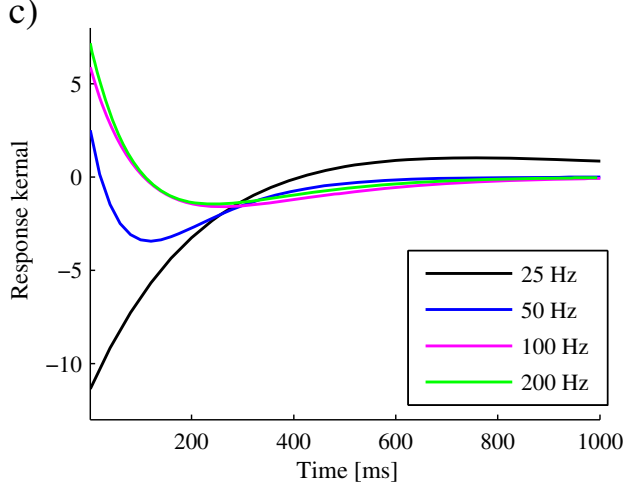


experimental data with better accuracy than the autocorrelation function of the model without the response kernel (the black and red traces and similar, while the blue trace is not).

Figure 7 shows the model predictions vs. recorded spike trains for six different pulse frequencies. For each frequency, red dots indicate an experimentally recorded spike, blue lines give the predicted spike probability for that pulse, and black dots give a stochastically predicted spike train based on the spike probability. While there are some overestimated spikes, in general the model predicts higher probability of spiking when the spike is recorded and lower probability of spiking during the suppression time.

The static nonlinearity of the model, $G$, for $25,50,100$, and $200 \mathrm{~Hz}$ pulse rates for a sample cell is plotted in Fig. 8a. These simulations were based on the experimental data from one cell stimulated at different frequencies. The nonlinearity function, $G$, shows a different threshold for spiking when the cell is stimulated at different frequencies (note the long range of the x-axis). The response kernel $h$ for 25, 50, 100, and $200 \mathrm{~Hz}$ pulse rates is plotted in Fig. 8b, c. Note that $h$ has different time constants and amplitudes for the same cell for different frequencies of stimulation.

The values for the coefficients of variation of the interspike-interval with stimulation Protocol 1 are given in Fig. 9a. Simulations results show the average for ten stochastic iterations for each experimentally recorded spike train. Experimental data is averaged for each frequency of stimulation. Results show that for all stimulation frequencies, the difference between the coefficient of variation with the spike history model and the experimental data is smaller than the difference between the coefficient of variation with the model without response kernel and the experimental data. Note the higher coefficient of variation for higher frequencies of stimulation. This is due to a cluster-suppression phenomenon at higher frequencies.

The amplitude of the history kernel for results with Protocol 1 are given in Fig. 9b. The values, $A_{1}+A_{2}$, are shown by red stars for different frequencies of stimulation. The results show that for $25 \mathrm{~Hz}$ stimulation the kernel has negative amplitude, for $50-200 \mathrm{~Hz}$ stimulation the kernel has positive amplitude, and for $500-100 \mathrm{~Hz}$ stimulation the kernel has negative amplitude. Note, the negative value of the history kernel at $500-100 \mathrm{~Hz}$ stimulation may be due to relatively high amplitude of the stimulation used in Protocol 1. Black stars indicate the amplitude of the opposite phase reflection of the kernel (found with minimal values for 25 and $200 \mathrm{~Hz}$ stimulations only).

Figure 10 illustrates the Victor-Purpura metric (VP Metric), the frequency-scaled metric $E_{\Delta}$, the metric based on the spike times (Spike Time Metric), and the metric based on the inter-spike intervals (ISI Metric). The results for all cells stimulated with Protocol 1 were averaged for each frequency of the stimulation. Ten stochastic simulations were run for each experimentally recorded spike train and the results were averaged. Results show that the model with the spike history (black diamonds) approximates the experimental data better using all metrics (i.e., the penalty cost is lower). The VP and frequency-scaled metrics vary with the frequency of the stimulation, while the ISI metric decreases with the frequency of the stimulation. The higher penalty cost of the model without the response kernel (blue diamonds) is mostly due to extra spike insertions during the time of suppression in experimentally recorded spike train.

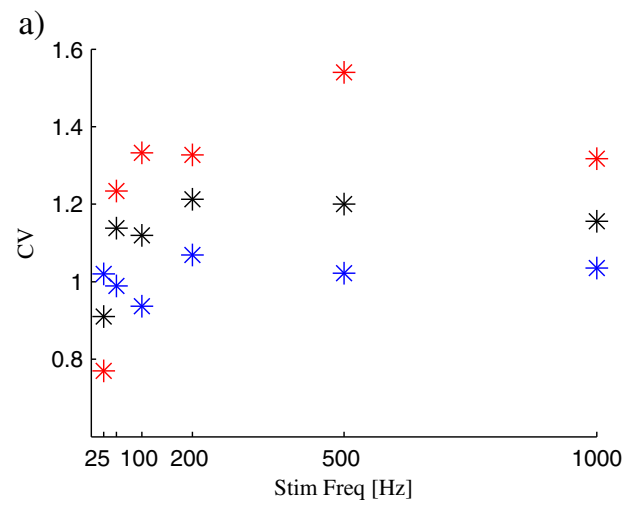

Fig. 9 Ten stochastic simulations were run for each experimentally recorded spike train and the results were averaged. a) The coefficient of variation of the inter-spike interval as a function of stimulation frequency with Protocol 1. Red stars: experimental data. Black stars: the model with the history kernel. Blue stars: the model without the history b)

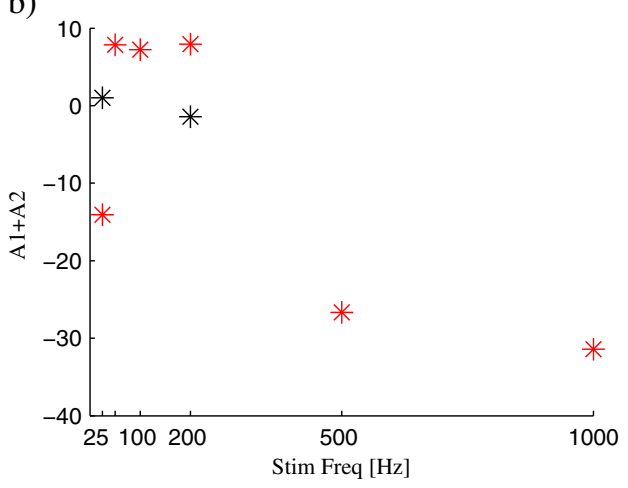

kernel. b) The amplitude of the history kernel, $A_{1}+A_{2}$, with stimulation Protocol 1 (red stars). Black stars: the amplitude of the opposite phase reflection of the kernel. a), b) Experimental data averaged for each stimulation frequency. $50 \mathrm{~Hz}$ stimulation frequency is indicated by a tick only for better visualization 

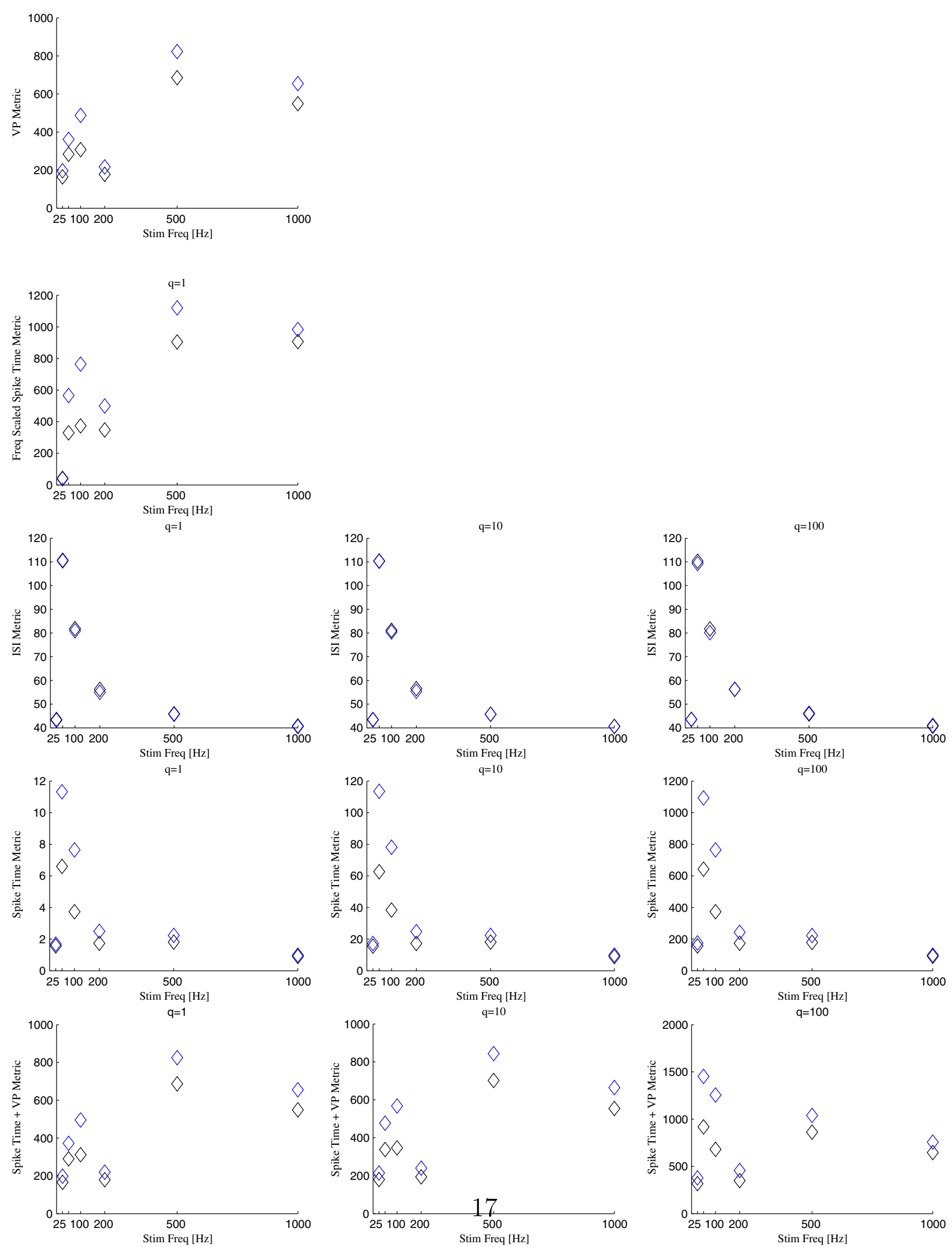

Fig. 10 The Victor-Purpura metric (VP Metric), the frequency-scaled spike time metric $\left(E_{\Delta}\right)$, the metric based on the spike times (Spike Time Metric), and the metric based on the inter-spike intervals (ISI Metric). The value of the parameter $q$ used in simulations is shown above the plots. The metrics for the model with the spike history are shown in black and for the model where the spike history is neglected in blue. Simulations were ten stochastic iterations for each experimentally recorded spike train. The results are averages for each frequency of the stimulation for all cells. $50 \mathrm{~Hz}$ stimulation frequency is shown by a tick only 


\subsection{Stimulation protocol 2}

\subsubsection{Experiments}

Figure 11a illustrates the response probability for the experimental data given the stimulation level for stimulation Protocol 2. All cells responded to both cathodic-first and anodic-first pulses. In all experiments, the difference between anodic-first and cathodic-first thresholds was less than $10 \%$. The response probability for experimental data plotted as a function of stimulation value, is shown in Figs. 11a. The response probability for experimental data, given a generator signal value, $x$ in Eq. (3), is shown in Fig. $11 \mathrm{~b}$ by red stars. The function is plotted as discrete stars since the experimental data do not contain the continuum of stimulation values. The response probability in Fig. 11a and $b$ was averaged over 10 repetitions with the same stimuli.

The internal neuron's response variability for the same stimulation train applied repeatedly to a cell is shown in Fig. 12 by red dots.

\subsubsection{Modeling}

Predicted response probability using the spike history model (1) is illustrated in Fig. 11b by a solid black line. Note how well the model predicts experimentally recorded data.

To test ability of the model to predict spikes and to examine the variability in the response and compare it to the model variability, we stimulated the same cell up to 10 repetitions with $200 \mathrm{~Hz}$ pulse rates, 5000 pulses in each repetition. Figure 12 shows the model predictions vs. recorded spike trains. Experimentally recorded spikes are given by red dots, and spike history model (1) predictions are given by black dots (for ten simulation runs), the model predictions without the response kernel (8) are given by blue dots (for ten simulation runs). Both models robustly predict the experimentally recorded spike-train; however, the model with history $(h \neq 0)$ better predicts the spike suppressions. A model with the response kernel produces less spikes (less black dots) than the model without the response kernel (blue dots) during the periods of silence recorded experimentally. This is also quantified by the penalty values in metrics illustrated in Fig. 10.

The Victor-Purpura (VP) metric, the frequency-scaled spike time metric $E_{\Delta}$, the metric based on the spike times, and the metric based on the inter-spike intervals for repetitions of $200 \mathrm{~Hz}$ stimulation with Protocol 2 are given in Table 1 . The superscript $h$ denotes the values for the spikehistory model, the superscript $h$ denotes the values for the model where the spike history is neglected. The values given are the averages of ten stochastic simulations for each experimentally recorded spike train, averaged for each frequency of the stimulation. The results show that the model with the response kernel has a smaller penalty cost (the values of all metrics are smaller) than the model where the spike history is neglected. The coefficient of variation (CV) of the model with the history kernel closer approximates the $\mathrm{CV}$ of the data than the model without the history kernel. Results show that for all cells stimulated with $200 \mathrm{~Hz}$ the response kernel has positive amplitude $\left(A_{1}+A 2>0\right)$ with almost no opposite phase $(\min (h))$. (Fig. 13)

\subsection{Example: spike history model for neural control}

In this section, we provide a simulation example of how the spike history model can be used to control excitation of retinal ganglion cells.

For this example, we re-write Eqs. (1)-(4) in the following form:

$P_{t}=\frac{1+\tanh \left[a s_{t-1}+b\left|s_{t-1}\right|+\left(A_{1} e^{-t / \tau_{1}}+A_{2} e^{-t / \tau_{2}}\right) \cdot r_{\left[t-t_{1}, t-1\right]}-d\right]}{2}$.

The calculations below were performed with parameters obtained in one of the simulations above: $a=0.022, b=$ $0.11, d=5.61, A_{1}=440, A_{2}=-268, \tau_{1}=0.021, \tau_{2}=$ 0.037 .

In order to design a controller for the system (11), we take advantage of the fact that the system can be decomposed
Fig. 11 Red stars: a response probability for the experimental data given a) the stimulation level, and $\mathrm{b}$ ) a generator signal value, $x$. The calculated response probability was averaged over 10 repetitions with the same stimuli. Solid line: predicted response probability using the spike history model (1)
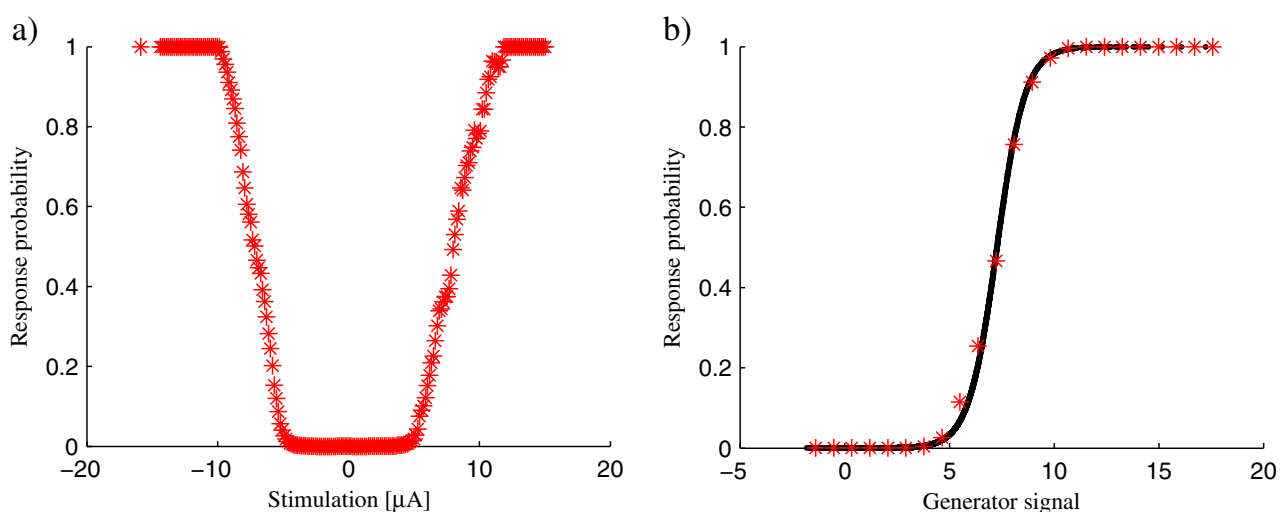


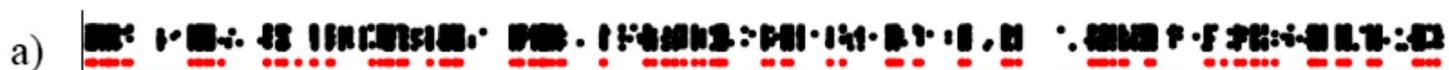

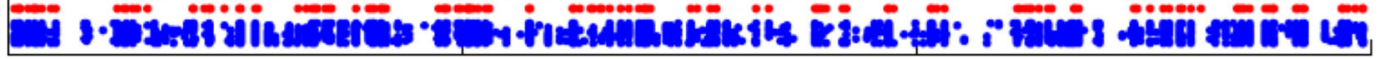
$\begin{array}{lrrr}0 & 500 & 1000 & 1500\end{array}$

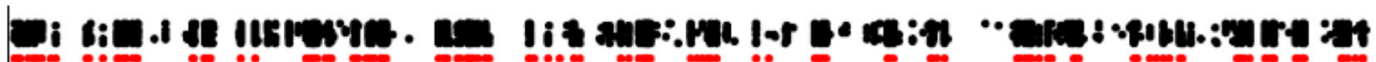

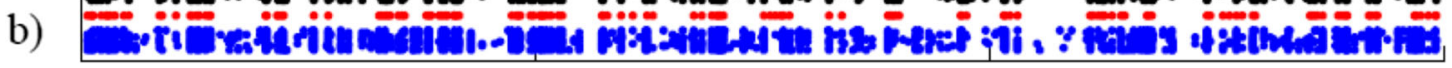
$\begin{array}{llll}0 & 500 & 1000 & 1500\end{array}$

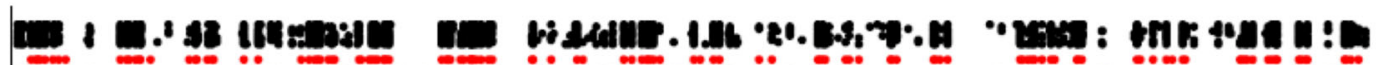

c) L-1. $\begin{array}{lllr}0 & 500 & 1000 & 1500\end{array}$

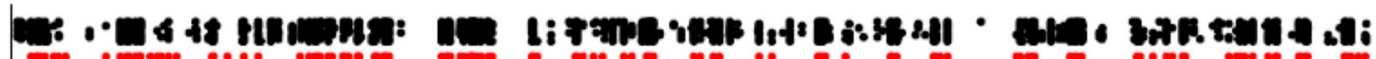

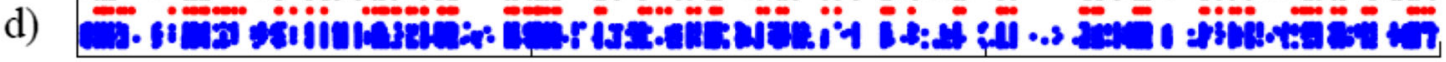
$\begin{array}{lrrr}0 & 500 & 1000 & 1500\end{array}$

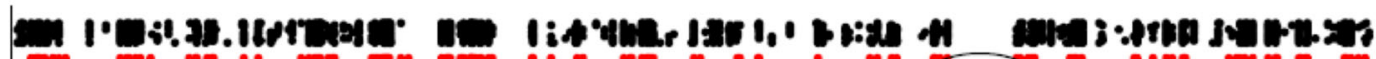

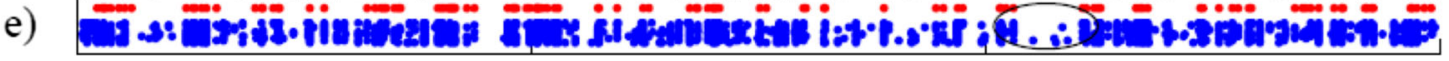
$\begin{array}{lrrr}0 & 500 & 1000 & 1500\end{array}$

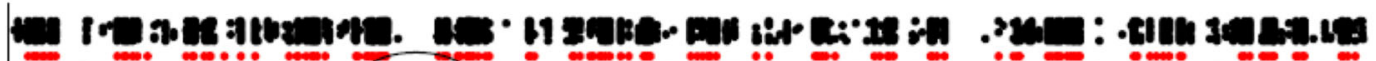

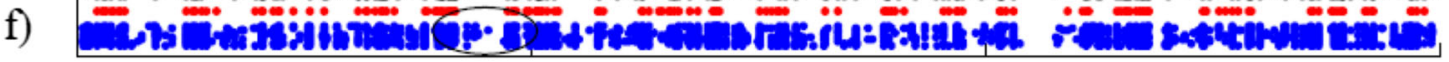
$\begin{array}{lrrr}0 & 500 & 1000 & 1500\end{array}$

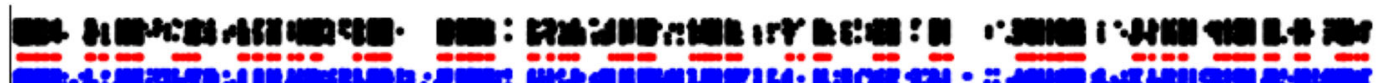

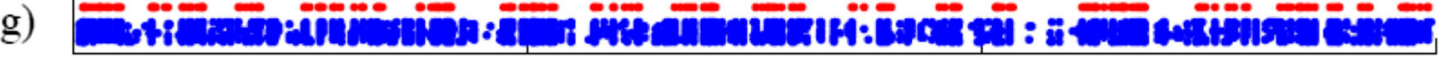
$\begin{array}{lrrr}0 & 500 & 1000 & 1500\end{array}$

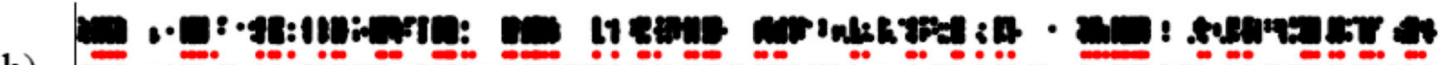

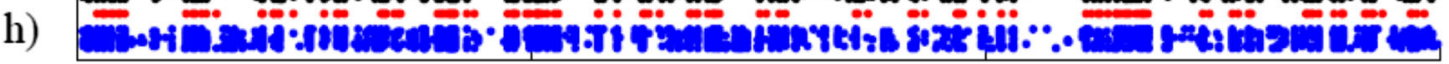
$\begin{array}{llll}0 & 500 & 1000 & 1500\end{array}$

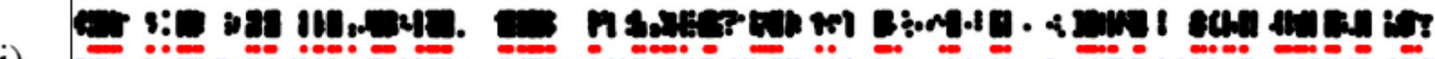

i) $\begin{array}{lrrr}0 & 500 & 1000 & 1500\end{array}$

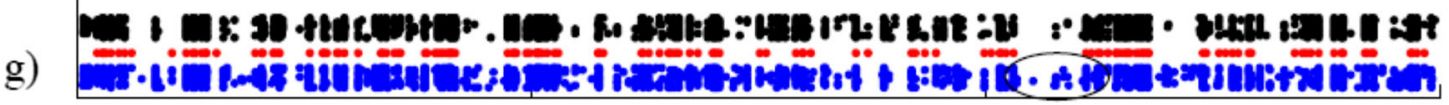
$\begin{array}{llll}0 & 500 & 1000 & 1500\end{array}$

Pulse \#

Fig. 12 A raster plot for several repetitions for a cell with the repeated stimuli at $200 \mathrm{~Hz}$. The repetitions are shown in subplots a) to l). Experimentally recorded spikes are given by red dots. Ten stochastically predicted trains for each of the repetition are shown by black and blue dots; spike history model predictions are given by black dots, the

into a sum of the static and dynamic parts followed by the nonlinearity, a sigmoid $G$. The static part is

$S=a s_{t-1}+b\left|s_{t-1}\right|$,

and the dynamic part is

$D=\left(A_{1} e^{-t / \tau_{1}}+A_{2} e^{-t / \tau_{2}}\right) \cdot r_{\left[t-t_{1}, t-1\right]}$.

To convert the probability of spiking, $P_{t}$, into a spike train of zeros (no spike) and ones (spike), a relay block is added, model predictions without the response kernel, $h=0$ in (1), are given by blue dots. Ovals show sample of periods in which the model without the response kernel does not reproduce spike suppression observed in the experimental data

as shown in Fig. 14. Below we describe how the outputs of the blocks in Fig. 14 are calculated.

Static system block The output of the static system, $y_{1}$, depends only on the stimulus amplitude,

$y_{1}=a s+b|s|=\left\{\begin{array}{l}s(a+b) \text { if } s \geq 0 \\ s(a-b) \text { if } s<0 .\end{array}\right.$

A graphical representation of the output of the static system given the stimulus amplitude, s, is given in Fig. 15a. 
Table 1 Metrics for simulated spike train with Protocol 2 for repetitions of $200 \mathrm{~Hz}$ stimulation

\begin{tabular}{|c|c|c|c|}
\hline CV Data & 1.77 & & \\
\hline $\mathrm{CV}^{h}$ & 1.23 & & \\
\hline $\mathrm{CV}^{\not h}$ & 1.04 & & \\
\hline$A_{1}$ & 81.70 & & \\
\hline$A_{2}$ & -18.05 & & \\
\hline $\min (h)$ & -1.94 & & \\
\hline$A_{1}+A_{2}$ & 63.64 & & \\
\hline VP Metric ${ }^{h}$ & 573 & & \\
\hline \multirow[t]{2}{*}{ VP Metric ${ }^{h /}$} & 675 & & \\
\hline & $q=1$ & $q=10$ & $q=100$ \\
\hline Spike Time Metric ${ }^{h}$ & 4 & 49 & 490 \\
\hline Spike Time Metric ${ }^{h /}$ & 7 & 74 & 739 \\
\hline ISI Metric ${ }^{h}$ & 110 & 110 & 110 \\
\hline ISI Metric ${ }^{\not /}$ & 109 & 109 & 109 \\
\hline Spike Time + VP Metric ${ }^{h}$ & 577 & 622 & 1063 \\
\hline Spike Time + VP Metric ${ }^{\not /}$ & 682 & 749 & 1414 \\
\hline
\end{tabular}

Dynamic system block To obtain the state-space equation for the dynamic system, we first write the transfer function for this system and then convert it into a time domain linear state-space representation with state $x$, input $r$, and output $y_{2}$ :

$\left\{\begin{array}{l}x(n+1)=A x(n)+B r(n) \\ y_{2}(n)=C x(n)\end{array}\right.$

where $n$ are sampling instances,

$A=\left[\begin{array}{cc}-74.6 & -1287 \\ 1 & 0\end{array}\right], B=\left[\begin{array}{l}1 \\ 0\end{array}\right], C=\left[\begin{array}{ll}1400 & -15701\end{array}\right]$.

The step response for the dynamic system is given in Fig. $15 b$.

Nonlinearity block. Given the parameters of the system, the resulting nonlinearity of the response, $G$, for the full system is given in Fig. 15c.

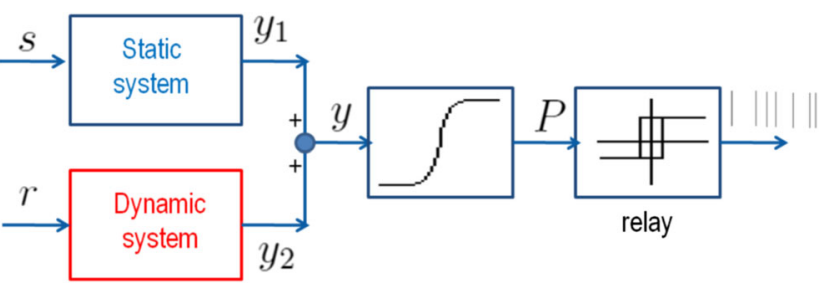

Fig. 14 Graphic representation of the system (11) as a sum of static, $y_{1}$, and dynamic, $y_{2}$, parts followed by the nonlinearity, $G$ (taken here as a tanh-function), and a relay block to convert the probability of spiking into a train of zeros and ones

Relay block. To set the parameters of the relay block, we need to consider the following. Assume that $y_{2}$ is constant (this assumption is relaxed later) and a spike is generated if $G \geq 0.5$ (this corresponds to $\tanh (x)>0$, that is, $\left.y_{1}+y_{2}+d \geq 0\right)$. Given the amplitude of the first phase of the pulse stimulation, $s \geq 0$ or $s<0$, it is possible that a spike was elicited $(r=1$, correspond to $\tanh (x)>0)$ or not $(r=0$, correspond to $\tanh (x)<0)$. This leads to four cases:

Case 1. $r=1, s \geq 0$.

In this case, we have:

$\left\{\begin{array}{l}y_{1}+y_{2} \geq d \\ y_{1}=s(a+b)\end{array} \Rightarrow s \geq \frac{d-y_{2}}{a+b}=l_{2}\right.$.

Case 2. $r=1, s<0$.

In this case, we have:

$\left\{\begin{array}{l}y_{1}+y_{2} \geq d \\ y_{1}=s(a-b)\end{array} \Rightarrow s<\frac{d-y_{2}}{a-b}=l_{1}\right.$.

Case 3. $r=0, s \geq 0$.

In this case, we have:

$\left\{\begin{array}{l}y_{1}+y_{2}<d \\ y_{1}=s(a+b)\end{array} \Rightarrow s<\frac{d-y_{2}}{a+b}=l_{2}\right.$.

Case 4. $r=0, s<0$.

In this case, we have:

$\left\{\begin{array}{l}y_{1}+y_{2}<d \\ y_{1}=s(a+b)\end{array} \Rightarrow s \geq \frac{d-y_{2}}{a-b}=l_{1}\right.$.

Fig. 13 Nonlinearity and response kernel for ten repetitions for the cell with the same stimuli at $200 \mathrm{~Hz}$. a) Nonlinearity $G$, calculated for different frequencies of stimulation for the same cell, plotted as a function of generator signal, $x . \mathrm{b})$ Response kernel, $h$, calculated for different frequencies of stimulation for the same cell, plotted as a function of pulse number a)

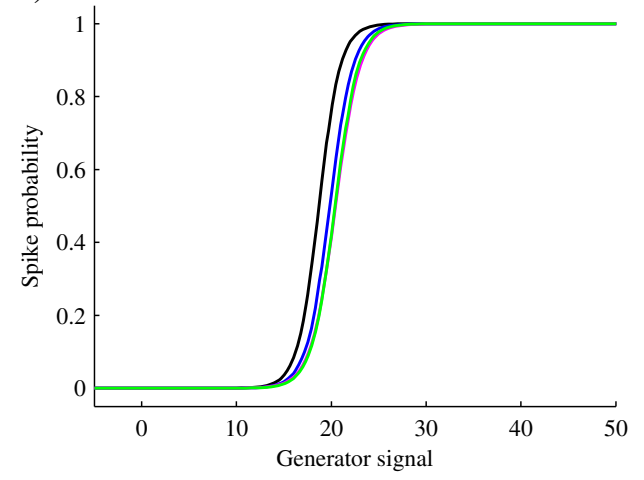

b)

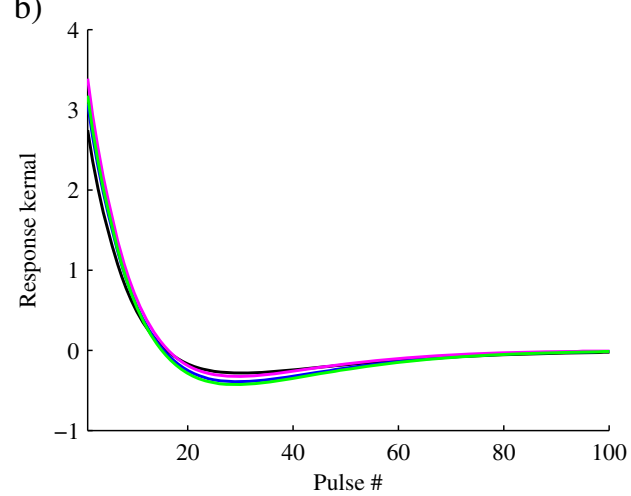


Fig. 15 a) Static system.

Graphical representation of the output of the static system, $y_{1}$, given the amplitude of the stimulus, $s$. b) Dynamic system. Step response, $h$, for the dynamic system. c) Nonlinearity, $G$, for the full system. d) Relay block; $l_{1}, l_{2}$ are calculated based on (16)- (19) a)

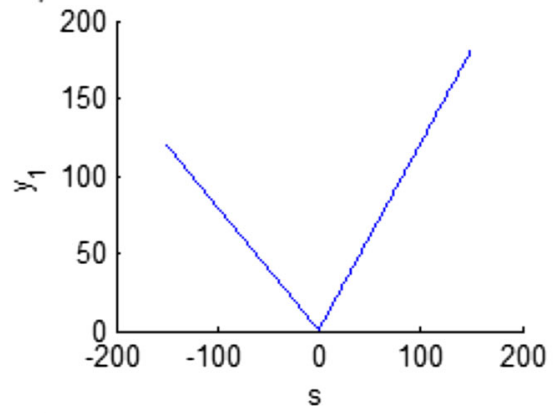

c)

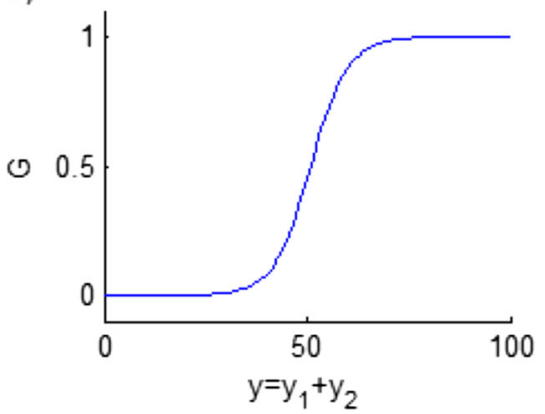

b)

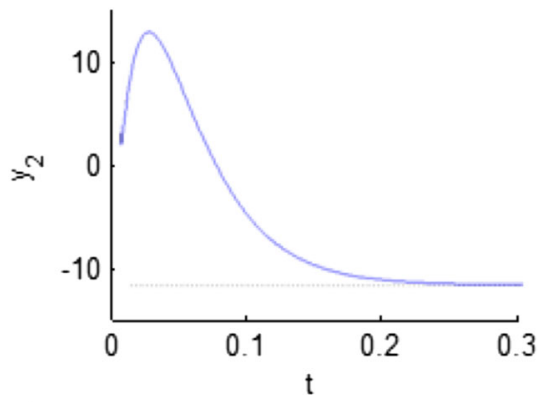

d)

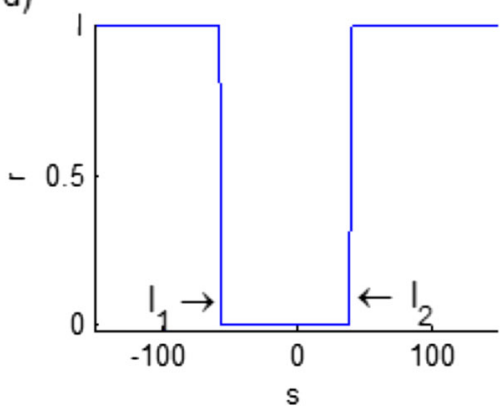

In other words, a spike is elicited if the amplitude of the first phase of the stimulus pulse $s \geq l_{2}>0$ or $s<l_{2}<$ 0 . If the value is set between $l_{1}$ and $l_{2}$, then a spike is not elicited. The relay that converts the probability function, $G$, into a spike train using the parameters above is shown in Fig. 15d.

The analysis above was performed under the assumption that the output of the dynamic system $y_{2}$ is constant. The probability of the spike given different values of $y_{2}$ and the amplitude of the stimulus, $s$, is shown in Fig. 16.

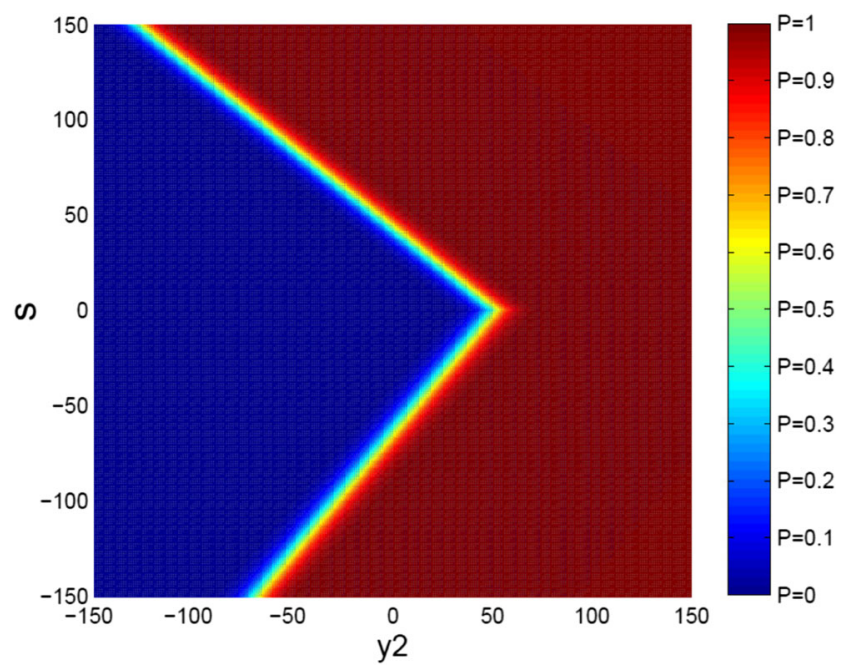

Fig. 16 Probability of spiking, $G$, for different values of the output dynamic system, $y_{2}$, and stimulus amplitude, $s$

\subsubsection{Open-loop controller}

A block diagram of the open-loop controller is given in Fig. 17. To set the stimulus amplitude using the open-loop controller, consider the following. If the reference is zero (no spike), then the amplitude is set to zero, $s=0$. If the reference is one (a spike), then the amplitude of the stimulus, $s$, is chosen based on the desired probability to elicit a spike. When the system is an open loop, the state and the output of the system are not known; therefore, we cannot use a recorded spike train, $r$, for the controller design. To estimate the dynamic part of the system, $y_{2}$, conservatively, we set it to the minimum possible value, -12.2. This gives the lowest probability of spiking.

The amplitude of the stimulus given a desired probability is given in Fig. 18. For example, if the reference is 1 (spike) and the desired probability to elicit a spike is 0.95 , then the amplitude of the stimuli is set to $64 \mu \mathrm{A}$. The amplitude of stimulation using the open-loop controller is illustrated in Fig. 20a. Errors between the reference spike train and simulated train using open-loop controller are shown in Fig. 20c; over five seconds stimulation there were only five errors.

\subsubsection{Closed-loop controller}

A block diagram of the closed-loop controller is given in Fig. 19. Similar to the open-loop system, if the reference is zero (no spike), then the amplitude is set to zero, $s=$ 0 . If the reference is one (a spike), then the amplitude of the stimulus, $s$, is set based on the desired probability to 
Fig. 17 A block diagram of the open-loop system. The recorded spike train signal, $r$, is not fed back into the controller; therefore, the controller action, $s$, does not depend on the output of the system

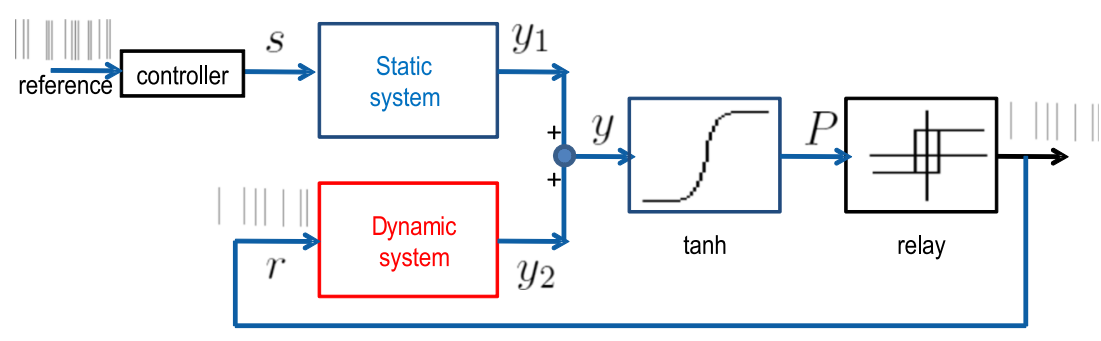

elicit a spike. In the closed-loop case, the measurement of the output of the system, $r$, is fed back into the controller. Therefore, the stimulus amplitude depends on the history of the neuron's spiking.

The amplitude of stimulation using the closed-loop controller is shown in Fig. 20b; the errors between the closedloop system simulated spike train and the reference signal are illustrated in Fig. 20d.

\subsubsection{System with disturbances}

To simulate system with disturbances, a uniformly distributed noise on the interval $[0,1]$ was introduced to the spike rate measurements, $r(t)$, that corresponded to the measurement disturbances. In addition to that, a uniformly distributed noise on the interval $[0,1]$ multiplied by the constant coefficient $5 \times 10^{-3}$ was added to the sigmoid block of the system. This noise corresponded to the model uncertainties.

Figure $21 \mathrm{~b}$ illustrates the results when disturbances are introduced into the system. The amplitude of stimulation using the closed-loop controller is given in Fig. 21b; the errors between the closed-loop system simulated spike train and the reference signal are given in Fig. 21d. While the open-loop controller performs well, the amplitude of the stimuli using the closed-loop controller is smaller than when

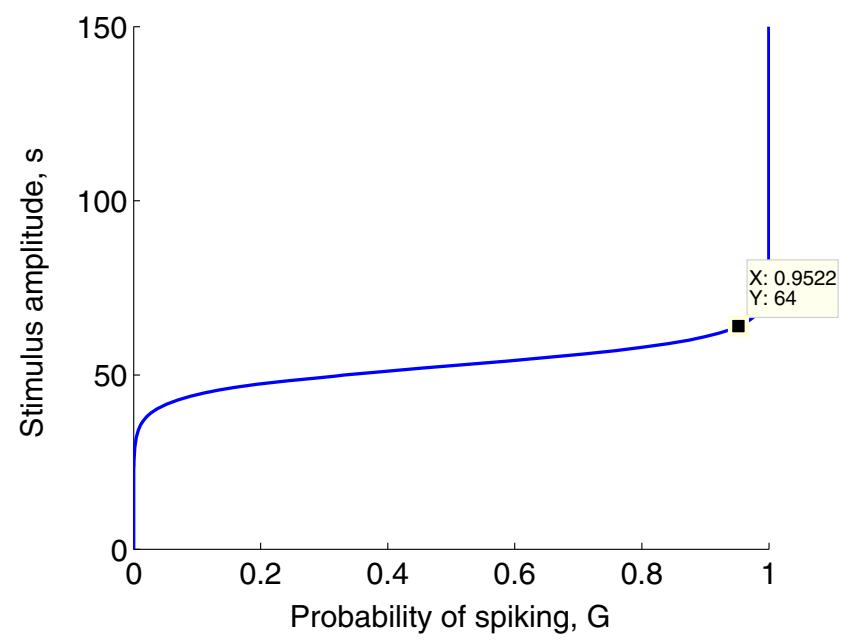

Fig. 18 Open-loop setup. The amplitude of the stimulus given a desired probability. See text for details using open-loop controller. Note that the feedback controller shows much better performance. We show that with larger noise amplitude, the difference between feedback and feedforward controllers will be even larger.

\section{Discussion and conclusions}

In this study, we presented a simple model of the retinal ganglion cell responses to electrical stimulation. This model can be used in neuroprosthetic devices that incorporate modelbased controller design. The model parameters were fitted based on the collected experimental data from rabbit retinae in vitro. Our simulations show that using closed-loop stimulation, it is possible to save power. Our results show that while the open-loop controller performs well, the amplitude of the stimuli using the closed-loop controller is smaller than when using open-loop controller, leading to considerable power savings. Similar results were observed in a study by Gorzelic et al. (2013) that investigated optimization techniques for a model-based proportional-integrate-derivative controller in application to Parkinson's disease.

It is likely, that the electrode-neural interphase will change over time and the stimulation parameters will need to be re-tuned. This phenomenon is well known from the cochlear implant users (for review, see (Clark 2003)). Similarly, the visual implants users will need to re-adjust stimulation parameters due to the retinal network remodeling during degeneration (Mark et al. 2003). The network remodeling, fiber-tissue growth, and the electrode-tissue interphase change will introduce deviations to the neural response models. We have shown that the model uncertainties and measurement disturbances are best dealt with using the closed-loop stimulation strategies.

Our experimental data demonstrated clusters of spikes followed by periods of suppression in spike trains above 100 $\mathrm{Hz}$ stimulation. The phenomenon of cluster-suppression with white-noise stimulation may be indicative of memory in the system; i.e., the response depends not only on the current stimulus but also on the responses within a time window preceding the current time. To confirm this, we calculated the auto-correlation function of the recorded spike trains for different frequencies of stimulation. This showed that there was memory in the system for stimulation at frequencies 
Fig. 19 A block-diagram of the closed-loop system. The estimated controller signal (the amplitude of the stimulation), $s$, depends on the recorded spike train, $r$. The inverse of the dynamic system is fed back into the controller

Fig. 20 Simulated spike train using open-loop and closed-loop controllers. a) Stimulation amplitude using open-loop controller. b) Stimulation amplitude using closed-loop controller. c) Errors between the reference spike train and simulated spike train using open-loop controller. c) Errors between the reference spike train and simulated spike train using closed-loop controller

Fig. 21 Simulated spike train using open-loop and closed-loop controllers when measurement disturbances and model uncertainties are introduced into the system. a) Stimulation amplitude using open-loop controller. b) Stimulation amplitude using closed-loop controller. c) Errors between the reference spike train and simulated spike train using open-loop controller. c) Errors between the reference spike train and simulated spike train using closed-loop controller

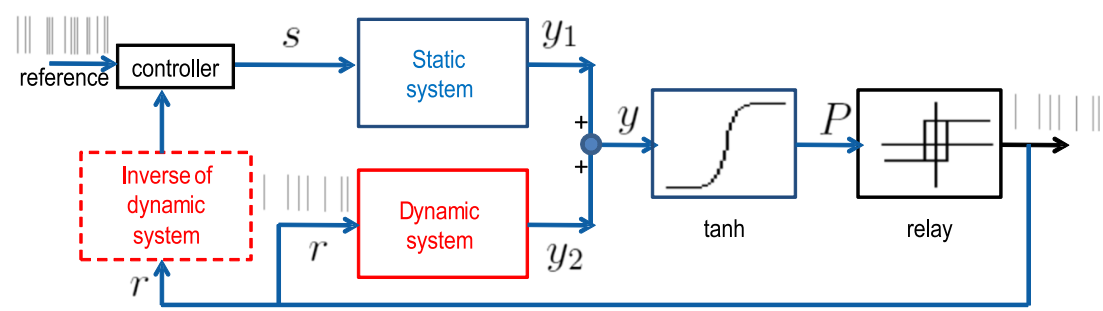

a)

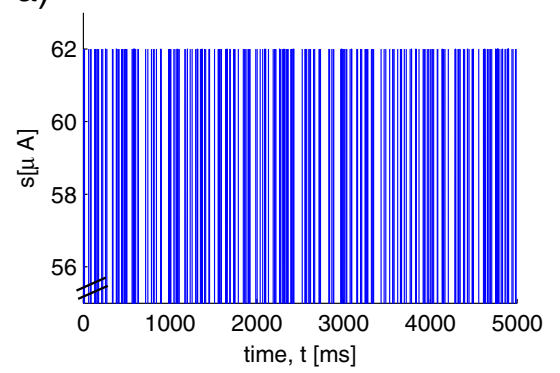

c)

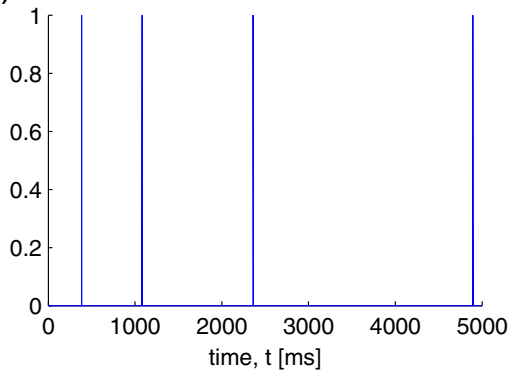

b)

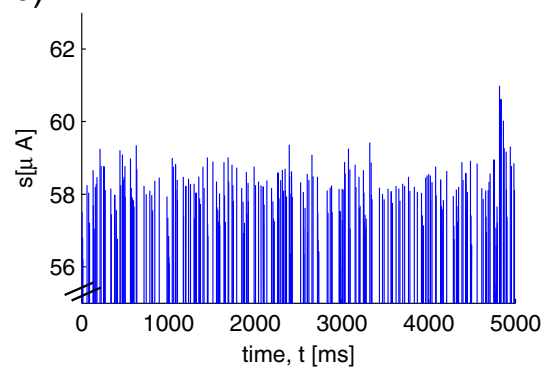

d)

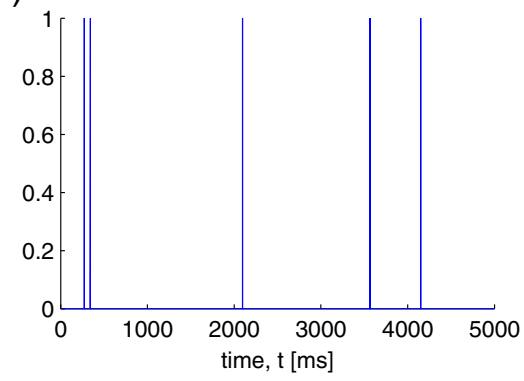

a)

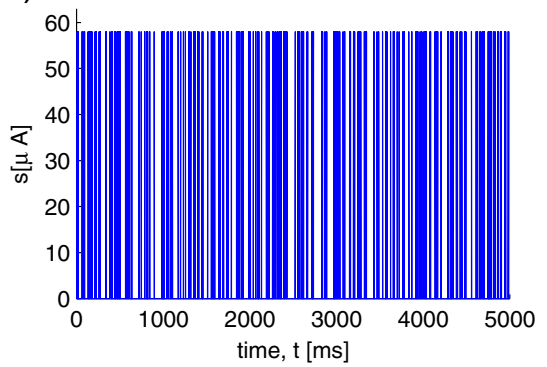

c)

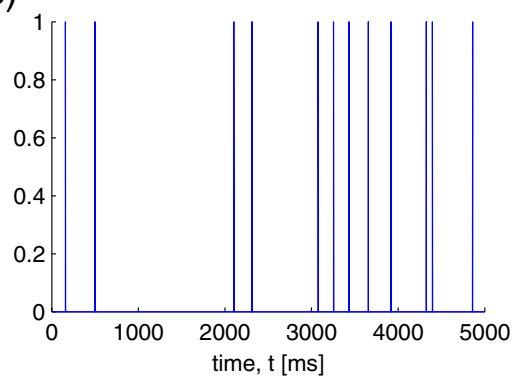

b)

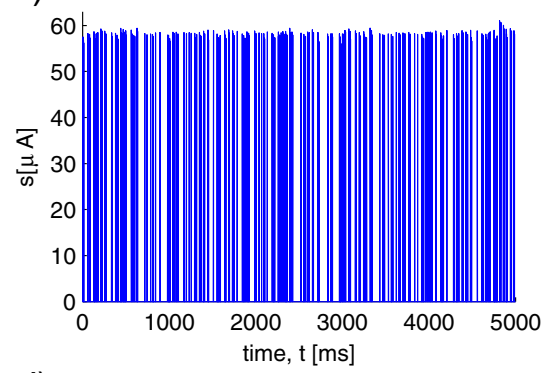

d)

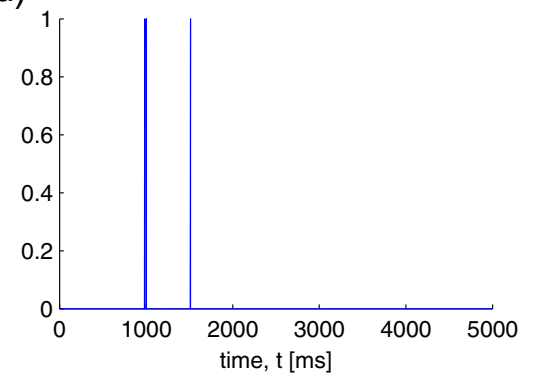


higher than $100 \mathrm{~Hz}$. Robustness of the model parameters was confirmed by repeated stimulation of the same cell at the same frequency (up to 10 repetitions). Comparison of the model with history and the model without history confirmed that the model with history approximates coefficient of variation of the experimental data better.

The phenomena of spike self-organization, cellular memory, and activity-dependent suppression of spontaneous spikes, were investigated by Rose et al. (2007), Sun et al. (2010), and Pulver and Griffith (2010). While the model proposed here was based on data from rabbit retinae, the methods developed here can be applied to data collected from other types of neurons to fit model parameters. While we observed some memory in the RGCs responses, its origin is unknown and requires further investigation.

This paper presents results for single cell modeling. This model can be generalized to account for network connections and to include spacial activation of neural tissue. RGCs were stimulated using epiretinal stimulation and direct cell responses were recorded. The modeling framework presented here can be extended to suprachoroidal or subretinal stimulation, in which case the current passes through the network of bipolar/amacrine neurons to activate RGCs synaptically.

The model presented here accounts for passive dynamic electrophysiology and static nonlinear electrophysiology since the linear-nonlinear model may be derived from the combined volume conductor (cable equation) model of electrical neural activation (see Rattay 1999; McNeal 1976) using a set of assumptions, i.e., the subthreshold dynamics of the membrane potential is modelled by a linear cable equation and the nonlinear spiking behavior is captured by a static nonlinearity. The spike history term presented here adds an additional level of the complexity to the model.

We found that the value of the amplitude of the response kernel is different for different frequency of stimulation. For some frequencies, the history kernel represents a fatigue effect, while for others the kernel shows a facilitatory effect. A combination of the ion channels and 3-dimensional structure of the cell can explore the origin of these mechanisms. These were not investigated here.

A recent publication by Jepson et al. (2014a) discussed the use of patterns of electrical stimulation to increase the spatial resolution of a visual prosthesis. The authors presented a linear-nonlinear model that predicts the neural response to electrical stimulation. Their model is similar to the framework presented here; however, the novel white noise stimuli and spike history proposed in our publication were not used by Jepson et al. (2014a). The method of white noise electrical stimulation proposed in our paper gives many advantages, including the possibility of predicting neural response based on a limited number of measurements.

The motivation for the model presented here comes from closed-loop stimulation strategies in neuroprosthetic devices. A memory in the system indicates that the closedloop strategy will be more beneficial to find an optimal stimulation level. It is reasonable to expect that the advantages of the feedback stimulation strategies will be more apparent when the feedback is implemented in vitro than compared to simulations results presented here, since measurement disturbances are much better accounted for by a well-designed closed-loop controller that by an open-loop controller.

Closed-loop stimulation requires simultaneous recording and stimulation using multiple electrodes array. Methods for multi-electrode recording, precise neuronal activation, and stimulation artifact removal are improving rapidly (Muller et al. 2013; Jepson et al. 2014a, b; Wallach et al. 2011).

Modern control theory has been developing since the 1950s. While it has brought many advances in industrial design (Goodwin et al. 2001), its available tools remain under-utilized in neuroscience (Schiff 2009). With the advances in computational power and improvements in medical bionic devices, there is a possibility for control theory tools and neuroscience to unite at the forefront of biomedical research and clinical implementation.

\section{Compliance with ethical standards}

All applicable international, national and institutional guidelines for the care and use of animals were followed. All procedures performed in studies involving animals were in accordance with the ethical standards of the Animal Care and Ethics Committee at the University of New South Wales.

Acknowledgments This research was supported by the Australian Research Council (DE120102210). The Bionic Ear Institute acknowledges the support it receives from the Victorian Government through its Operational Infrastructure Support Program. This research was supported by the Australian Research Council through its Special Research Initiative in Bionic Vision Science and Technology grant to Bionic Vision Australia. NICTA is funded by the Australian Government as represented by the Department of Broadband, Communications and the Digital Economy and the Australian Research Council through the ICT Centre of Excellence program.

Conflict of interests The authors declare that they have no conflict of interest 


\section{References}

Abbas, J.J., \& Chizeck, H.J. (1991). Feedback control of coronal plane hip angle in paraplegic subjects using functional neuromuscular stimulation. IEEE Transactions Biomedical Engineering, 38, 687698.

Dayan, P., \& Abbott, L.F. (2001). Theoretical neuroscience: computational and mathematical modeling of neural systems: MIT press.

Chichilnisky, E.J. (2001). A simple white noise analysis of neuronal light responses. Network: Computation in Neural Systems, 12, 199-213.

Clark, G. (2003). Cochlear implants: fundamentals and applications: Springer.

Cooper, H.R., \& Craddock, L.C. (Eds.) (2006). Cochlear implants: a practical guide: Wurr Publishers.

Fountas, K.N., Smith, J.R., Murro, A.M., Politsky, J., Park, Y.D., \& Jenkins, P.D. (2005). Implantation of a closed-loop stimulation in the management of medically refractory focal epilepsy. Stereotact. Functional Neurosurgery, 83, 153-158.

Gerstner, W., \& Kistler, W.M. (2002). Spiking neuron models: single neurons, populations, plasticity. Cambridge: Cambridge University Press.

Goodwin, G.C., Graebe, S.F., \& Salgado, M.E. (2001). Control Systems Design: Prentice Hall International.

Gorzelic, P., Schiff, S.J., \& Sinha, A. (2013). Model-based rational feedback controller design for closed-loop deep brain stimulation of Parkinson's disease. Journal of Neural Engineering, 026016.

Jepson, L.H., Hottowy P., Matheson K., Gunning, D.E., Dabrowski, W., Litke, A.M., \& Chichilnisky, E.J. (2014a). Spatially patterned electrical stimulation to enhance resolution of retinal prostheses. Journal of Neuroscience, 34(14), 4871-4881.

Jepson, L.H., Hottowy, P., Weiner, G.A., Dabrowski, W., Litke, A.M., \& Chichilnisky, E.J. (2014b). High-fidelity reproduction of spatiotemporal visual signals for retinal prosthesis. Neuron, 83, 87-92.

Kalia, S.K., Sankar, T., \& Lozano, A.M. (2013). Deep brain stimulation for Parkinson's disease and other movement disorders. Current Opinion, 26(4), 374-380.

Keat, J., Reinagel, P., Reid, R.C., \& Meister, M. (2001). Predicting every spike: a model for the responses of visual neurons. Neuron, 30, 803-817.

Koch, C., \& Segev, I. (1998). Methods in neuronal modeling: from ions to networks. MIT press.

Lesica, N.A., Jin, J., Weng, C., Yeh, C.I., Butts, D.A., Stanley, G.B., \& Alonso, J.M. (2007). Adaptation to stimulus contrast and correlations during natural visual stimulation. Neuron, 55, 479-491.

Mark, R.E., Jones, B.W., Watt, C.B., \& Strettoi, E. (2003). Neural remodeling in retinal degeneration. Progress Retinal Eye Research, 22(5), 607-655.

McNeal, D.R. (1976). Analysis of a model for excitation of myelinated nerve. IEEE Transactions on Biomedical Engineering, 23(4), 329337.

Muller, J., Bakkum, D.J., \& Hierlemann, A. (2013). Sub-millisecond closed-loop feedback stimulation between arbitrary sets of individual neurons. Frontiers in Neural Circuits, 6(121)!-11.

Nelson, T.S., Suhr, C.L., Freestone, D.R., Lai, A., Halliday, A.J., McLean, K.J., Burkitt, A.N., \& Cook, M.J. (2011). Closed-loop seizure control with very high frequency electrical stimulation at seizure onset in the GEARS model of absence epilepsy. Journal of Neural Systems, 21(2), 163-173.

Nirenberg, S., Bornash, I., Pillow, J.W., \& Victor J.D. (2010). Heterogeneous response dynamics in retinal ganglion cells: the interplay of predictive coding and adaptation. Journal of Neurophysiology, 103, 3184-3194.

Tukhlina, N., \& Rosenblum, M. (2008). Feedback suppression of neural synchrony in two interacting pPopulations by vanishing stimulation. Journal of Biological Physics, 34, 301314.

Parker, J., Karantonis, D., Single, P., \& Obradovic, M. (2012). Compound action potentials recorded in the human spinal cord during neurostimulation for pain relief. Pain, 153(3), 593601.

Pillow, J.W., Paninski, L., Uzzell, V.J., Simoncelli, E.P., \& Chichilnisky, E.J. (2005). Prediction and decoding of retinal ganglion cell responses with probabilistic spiking model. Journal of Neuroscience, 25(47), 11003-11013.

Pulver, S.R., \& Griffith, L.C. (2010). Spike integration and cellular memory in a rhythmic network from $\mathrm{Na} / \mathrm{K}$ pump current dynamics. Nature Neuroscience, 13, 53-59.

Rattay, F. (1999). The basic mechanism for the electrical stimulation of the nervous system. Neuroscience, 89(2), 335-346.

Rose, T., Gras, H., \& Horner, M. (2007). Activity-dependent suppression of spontaneous spike generation in the Retzius neurons of the leech Hirudo medicinalis L. Invertebrate Neuroscience, 6(4), 169-176.

Rosin, B., Slovik, M., Mitelman, R., Rivlin-Etzion, M., Haber, S.N., Israel, Z., Vaadia, E., \& Bergman H. (2011). Closed-loop deep brain stimulation is superior in ameliorating Parkinsonism. Neuron, 72(2), 370-384.

Schiff, S.J. (2009). Kalman meets neuron: the emerging intersection of control theory with neuroscience. Proceedings of the IEEE EMBS conference, $1-4$.

Sun, J.J., Kilb, W., \& Luhmann, H.J. (2010). Self-organization of repetitive spike patterns in developing neuronal networks in vitro. European Journal of Neuroscience, 1-11.

Stingl, et al. (2013). Artificial vision with wirelessly powered subretinal electronic implant alpha-IMS. Proceedings of the Royal Society: Biological Sciences, 280(1757), 20130077.

van Hateren, J.N., Ruttiger, L., Sun, H., \& Lee, B.B. (2002). Processing of natural temporal stimuli by macaque retinal ganglion cells. Journal of Neuroscience, 22(22), 9945-9960.

Victor, J.D., \& Purpura, K.P. (1997). Metric-space analysis of spike trains: theory, algorithms and application. Network: Computation in Neural Systems, 8, 127-164.

Vetter, A.H., Masani, K., Nakazawa, K., \& Popovic, M.R. (2010). Neural-mechanical feedback control scheme generates physiological ankle torque fluctuation during quiet stance IEEE. Transactions on Neural Systems Rehabililation Engineering, 18(1), 86-95.

Wallach, A., Eytan, D., Gal, A., Zrenner, C., \& Marom, S. (2011). Neuronal response clamp. Frontiers in Neuroengineering, 4, $1-10$.

Zimmermann, J.B., \& Jackson, A. (2014). Closed-loop control of spinal cord stimulation to restore hand function after paralysis. Frontiers in Neuroscience, 10, 3389. 


\section{University Library}

\section{- M M N E R VA A gateway to Melbourne's research publications}

Minerva Access is the Institutional Repository of The University of Melbourne

Author/s:

Kameneva, T;Abramian, M;Zarelli, D;Nesic, D;Burkitt, AN;Meffin, H;Grayden, DB

Title:

Spike history neural response model

Date:

2015-06-01

Citation:

Kameneva, T., Abramian, M., Zarelli, D., Nesic, D., Burkitt, A. N., Meffin, H. \& Grayden, D. B. (2015). Spike history neural response model. JOURNAL OF COMPUTATIONAL NEUROSCIENCE, 38 (3), pp.463-481. https://doi.org/10.1007/s10827-015-0549-5.

Persistent Link:

http://hdl.handle.net/11343/55192 